Decaimento dos autovalores de operadores integrais gerados por séries de potências 

SERVIÇO DE PÓS-GRADUAÇÃO DO ICMC-USP

Data de Depósito:

Assinatura:

\title{
Decaimento dos autovalores de operadores integrais gerados por séries de potências
}

\author{
Douglas Azevedo Sant’ Anna
}

Orientador: Prof. Dr. Valdir Antonio Menegatto

Tese apresentada ao Instituto de Ciências Matemáticas e de Computação - ICMC-USP, como parte dos requisitos para obtenção do título de Doutor em Ciências - Matemática. VERSÃO REVISADA

USP - São Carlos

Abril de 2013 
Ficha catalográfica elaborada pela Biblioteca Prof. Achille Bassi e Seção Técnica de Informática, ICMC/USP, com os dados fornecidos pelo(a) autor(a)

\begin{tabular}{|c|c|}
\hline \multirow[t]{3}{*}{ A994d } & $\begin{array}{l}\text { Azevedo, Douglas } \\
\quad \text { Decaimento dos autovalores de operadores } \\
\text { integrais gerados por séries de potências. / Douglas } \\
\text { Azevedo; orientador Valdir Antonio Menegatto. -- São } \\
\text { Carlos, } 2013 \text {. } \\
\quad 55 \mathrm{p} \text {. }\end{array}$ \\
\hline & $\begin{array}{l}\text { Tese (Doutorado - Programa de Pós-Graduação em } \\
\text { Matemática)-- Instituto de Ciências Matemáticas e } \\
\text { de Computação, Universidade de São Paulo, } 2013 .\end{array}$ \\
\hline & $\begin{array}{l}\text { 1. Decaimento de autovalores. 2. Operadores } \\
\text { integrais. 3. Núcleos representados por séries de } \\
\text { potências. I. Menegatto, Valdir Antonio, orient. II. } \\
\text { Título. }\end{array}$ \\
\hline
\end{tabular}


Às minhas meninas,

Michele e Bela... 



\section{Agradecimentos}

Agradeço a minha família pelo apoio e aos amigos pelo companheirismo. Ao meu orientador Valdir Menegatto, agradeço a confiança e o direcionamento para a confecção deste trabalho. Agradeço também aos membros do grupo de pesquisa de Análise Funcional Aplicada e a todos professores e funcionários do ICMC. Por fim, agradeço à Capes e FAPESP pelo suporte financeiro concedido. 

O principal objetivo deste trabalho é descrever o decaimento dos autovalores de operadores integrais gerados por núcleos definidos por séries de potências, mediante hipóteses sobre os coeficientes na série que representa o núcleo gerador.

A análise é implementada em duas frentes: inicialmente, consideramos o caso em que o núcleo está definido sobre a esfera unitária de $\mathbb{R}^{m+1}$, estendendo posteriormente a análise, para o caso da bola unitária do mesmo espaço. Em seguida, visando primordialmente o caso em que o núcleo está definido sobre a esfera unitária em $\mathbb{C}^{m+1}$, abordamos um caso mais geral, aquele no qual o núcleo está definido por uma série de funções $L^{2}(X, \nu)$-ortogonais, sendo $(X, \nu)$ um espaço de medida arbitrário. 

The main target in this work is to deduce eigenvalue decay for integral operators generated by power series kernels, under general assumptions on the coefficients in the series representing the kernel.

The analysis is twofold: firstly, we consider generating kernels defined on the unit sphere in $\mathbb{R}^{m+1}$, replacing the sphere with the unit ball in a subsequent stage. Secondly, we consider generating kernels defined on a general measure space $(X, \nu)$ and possessing an $L^{2}(X, \nu)$-orthogonal expansion there, an attempt to cover the case in which the kernel is defined on the unit sphere in $\mathbb{C}^{m+1}$. 

Introdução $\quad$ Xv

1 Preliminares 1

1.1 Resultados da análise clássica $\ldots \ldots \ldots$. . . . . . . . . . . . . . . . 1

1.2 Resultados da teoria da medida . . . . . . . . . . . . . . . . . . 7

1.3 Resultados da análise funcional . . . . . . . . . . . . . . . . . . . . . . 10

2 Operadores integrais gerados por séries de potências. $\quad 15$

$2.1 \quad$ Positividade de $\mathcal{K} \ldots \ldots \ldots \ldots \ldots$

2.2 Representação em série para o operador integral . . . . . . . . . . . . . 18

2.3 Autovalores dos operadores gerados por séries de funções. . . . . . . . . 20

3 Decaimento dos autovalores $\quad 25$

$3.1 \quad$ Núcleos em séries de potências sobre $S^{m} \ldots \ldots \ldots \ldots$. . . . . . 25

3.2 Núcleos em séries de potências sobre $B^{m}$. . . . . . . . . . . . . . . 30

3.3 Alguns exemplos $\ldots \ldots \ldots$. . . . . . . . . . . . . . . . . . . . 33

4 Núcleos definidos por expansões ortogonais. 39

4.1 Resultados gerais . . . . . . . . . . . . . . . . . . . 39

$4.2 \quad$ Decaimento baseado na ordem parcial de $\mathbb{Z}_{+}^{m+1} \ldots \ldots$. . . . . . . . . . 41

4.3 Dois exemplos . . . . . . . . . . . . . . . . . . . . . 44

$\begin{array}{lll}5 & \text { Considerações finais } & 47\end{array}$

\begin{tabular}{ll}
\hline Referências Bibliográficas & 51
\end{tabular}

$\begin{array}{ll}\text { Índice Remissivo } & 56\end{array}$ 

Sejam $m$ um inteiro positivo e $X$ um subconjunto não-vazio do espaço $\mathbb{R}^{m+1}$ ou $\mathbb{C}^{m+1}$, munido da medida de Lebesgue usual $\nu$ do espaço. Propriedades teóricas e numéricas de operadores integrais limitados $\mathcal{K}: L^{2}(X, \nu) \rightarrow L^{2}(X, \nu)$ dados pela fórmula

$$
\mathcal{K}(f)=\int_{X} K(\cdot, y) f(y) d \nu(y), \quad f \in L^{2}(X, \nu)
$$

onde $K: X \times X \rightarrow \mathbb{C}$ é escolhido convenientemente, têm sido objeto de investigação em vários trabalhos recentes, como pode ser ratificado em [12, 18, 19, 20, 31, 32, 56, 58] e em outras referências citadas nestes trabalhos.

Em geral, o foco na obtenção de resultados deste tipo é investigar e descrever propriedades do operador integral, a partir de hipóteses previamente exigidas no núcleo gerador $K$. Ou seja, estabelecidas as hipóteses básicas sobre $K$ de modo que $\mathcal{K}$ esteja bem definido, analisar o efeito de propriedades adicionais sobre $K$ no operador $\mathcal{K}$. Particularmente, sob hipóteses convenientes, obter informações sobre o o espectro de $\mathcal{K}$, incluindo-se aqui, o comportamento assintótico dos autovalores ou valores singulares de $\mathcal{K}$.

No caso em que $K$ é um elemento de $L^{2}(X \times X, \nu \times \nu)$, o operador $\mathcal{K}: L^{2}(X, \nu) \rightarrow$ $L^{2}(X, \nu)$ é compacto. Além disso, se $K$ é hermitiano, ou seja, $K(x, y)=\overline{K(y, x)}, x, y \in$ $X$, o operador $\mathcal{K}$ é também autoadjunto. Assim sendo, o Teorema de Hilbert-Schmidt para operadores compactos e autoadjuntos garante uma representação $L^{2}(X, \nu)$-convergente da forma

$$
\mathcal{K}(f)=\sum_{n=0}^{\infty} \lambda_{n}(\mathcal{K})\left\langle f, \phi_{n}\right\rangle_{2} \phi_{n}, \quad f \in L^{2}(X, \nu)
$$

Aqui, $\left\{\lambda_{n}(\mathcal{K})\right\}$ é uma sequência de números reais (possivelmente finita) decrescente para 0 em valor absoluto e $\left\{\phi_{n}\right\} \subset L^{2}(X, \nu)$ é uma base ortonormal com relação ao 
produto interno usual de $L^{2}(X, \nu)$

$$
\langle f, g\rangle_{2}=\int_{X} f(x) \overline{g(x)} d \nu(x), \quad f, g \in L^{2}(X, \nu) .
$$

Consequentemente, os números $\lambda_{n}(\mathcal{K})$ são autovalores de $\mathcal{K}$ e a sequência $\left\{\lambda_{n}(\mathcal{K})\right\}$ já inclui as possíveis repetições impostas pela multiplicidade algébrica de cada um deles. Neste contexto, demonstra-se que ([34])

$$
K=\sum_{n=0}^{\infty} \lambda_{n}(\mathcal{K}) \phi_{n} \otimes \overline{\phi_{n}},
$$

onde $\phi_{k} \otimes \overline{\phi_{k}}(x, y):=\phi_{k}(x) \overline{\phi_{k}(y)}, x, y \in X$, e a convergência da série acima é em $L^{2}(X \times X, \nu \times \nu)$. Em particular, já temos garantido o decaimento básico $\left|\lambda_{n}(\mathcal{K})\right|=$ $o\left(n^{-1 / 2}\right)$, quando $n \rightarrow \infty$.

Adicionando-se ao contexto a continuidade e a positividade definida de $K$, o Teorema de Mercer ([15]) garante que cada coeficiente na representação acima é não negativo e que a convergência da série é uniforme nos subconjuntos compactos de $X$. Vale ainda a igualdade

$$
\int_{X} K(x, x) d \nu(x)=\sum_{n=0}^{\infty} \lambda_{n}(\mathcal{K})<\infty,
$$

quando $X$ é compacto, a qual implica na otimalidade do decaimento ([18, 50])

$$
\lambda_{n}(\mathcal{K})=o\left(n^{-1}\right), \quad(n \rightarrow \infty),
$$

nas condições descritas.

No caso em que $\mathcal{K}$ é compacto, mas não é autoadjunto, busca-se informações sobre os valores singulares de $\mathcal{K}$, ou seja, os autovalores do operador compacto, positivo e autoadjunto $|\mathcal{K}|:=\mathcal{K} \mathcal{K}^{*}: L^{2}(X, \nu) \rightarrow L^{2}(X, \nu)$.

Uma melhoria no decaimento básico mencionado acima demanda hipóteses adicionais ao núcleo gerador ou ao próprio operador $\mathcal{K}$. Isto foi, e ainda é, um tema de pesquisa em muitos trabalhos. No exemplo em que $X$ é um intervalo da reta real (ou mesmo a própria a reta), uma ampla investigação ocorreu ao longo do tempo, como pode ser verificado em [10, 11, 14, 30, 36, 41, 42, 43, 48, 49, 51]. Particularmente, G. Little ([42]) considerou núcleos positivos definidos possuindo representação em série de potências sobre $(-1,1)$, buscando informações sobre o decaimento dos autovalores a partir de certas hipóteses sobre os coeficientes presentes na representação do núcleo. No caso $X$ é um subconjunto de $\mathbb{R}^{m+1}$, as referências [38, 39, 40] analisaram o mesmo problema com os núcleos satisfazendo certas condições de Hölder. Em especial, V. Menegatto e colaboradores obtiveram êxito na análise do decaimento dos autovalores de $\mathcal{K}$, no caso em que $X$ é a esfera unitária de $\mathbb{R}^{m+1}$ e $K$ possui certa suavidade 
definida por condições de Lipschitz sobre as derivadas usuais de $K$ ([20]). Ainda no contexto esférico, em [12], decaimento para os valores singulares quando os núcleos são Laplace-Beltrami diferenciáveis foram obtidos.

Neste ponto, podemos concluir que a busca pela descrição do comportamento assintótico de autovalores do operador $\mathcal{K}$, mediante condições de suavidade sobre o núcleo gerador $K$, é um tema clássico na Análise Funcional ([23, 25, 29, 35]) o qual ainda mantém-se bastante ativo.

Neste trabalho, propomos investigar o decaimento dos autovalores do operador integral $\mathcal{K}$, no caso em que o núcleo gerador $K$ é dado por uma série de potências sobre $X$, isto é,

$$
K(x, y)=\sum_{\alpha \in \mathbb{Z}_{+}^{m+1}} a_{\alpha} x^{\alpha} \bar{y}^{\alpha}, \quad x, y \in X
$$

sujeito à condição

$$
\sum_{\alpha \in \mathbb{Z}_{+}^{m+1}}\left|a_{\alpha}\right||| p_{\alpha} \|_{2}^{2}<\infty
$$

mediante a exigências adicionais sobre os coeficientes $a_{\alpha}$. Aqui, $\|\cdot\|_{2}$ é norma usual em $L^{2}(X, \nu)$ enquanto que $p_{\alpha}(x)=x^{\alpha}=x_{1}^{\alpha_{1}} \ldots x_{m+1}^{\alpha_{m+1}}, x=\left(x_{1}, \ldots, x_{m+1}\right) \in X$ e $\alpha=$ $\left(\alpha_{1}, \ldots, \alpha_{m+1}\right) \in \mathbb{Z}_{+}^{m+1}$. O foco da análise aqui apresentada, visa cobrir o caso em que $X$ é a esfera unitária de $\mathbb{R}^{m+1}$ (ou $\mathbb{C}^{m+1}$ ), equipada com a medida de Lebesgue $\nu$ do espaço correspondente.

Nosso interesse na análise nesse contexto foi motivado pelo teorema multinomial clássico (Teorema 1.1.9). De fato, a descrição para $K$ acima inclui casos importantes já considerados na literatura: se "." e $\|\cdot\|$ indicam o produto interno e norma usuais de $\mathbb{R}^{m+1}, X=\left\{x \in \mathbb{R}^{m+1} ;\|x\| \leq R\right\}$ e $\sum_{n=0}^{\infty}\left|b_{n}\right| R^{2 n}<\infty$, para algum $R>0$, os núcleos polinomiais infinitos são da forma

$$
K(x, y)=\sum_{n=0}^{\infty} b_{n}(x \cdot y)^{n}=\sum_{\alpha \in \mathbb{Z}_{+}^{m+1}} b_{|\alpha|} \frac{|\alpha| !}{\alpha !} x^{\alpha} y^{\alpha}, \quad x, y \in X .
$$

Um ilustre exemplo nesta categoria é o famoso núcleo Gaussiano

$$
K(x, y)=\exp \left(-d\|x-y\|^{2}\right)=\exp \left(-d\left(\|x\|^{2}+\|y\|^{2}\right)\right) \sum_{\alpha \in \mathbb{Z}_{+}^{m+1}} \frac{(2 d)^{|\alpha|}}{\alpha !} x^{\alpha} y^{\alpha}, \quad x, y \in X .
$$

Este último tem sido amplamente investigado e utilizado em aplicações ([44, 55, 57, 64]).

A categoria dos núcleos definidos por séries de potências também inclui os núcleos não linearmente fatorizáveis. Esta categoria introduzida por B. Zwicknagl em [64], é composta por núcleos da forma

$$
K(x, y)=\prod_{k=1}^{m+1} f\left(x_{k} y_{k}\right)=\sum_{\alpha \in \mathbb{Z}_{+}^{m+1}}\left(\prod_{k=1}^{m+1} f_{\alpha_{k}}\right) x^{\alpha} y^{\alpha}
$$


Aqui, $f: \mathbb{R} \rightarrow \mathbb{R}$ é uma função conveniente possuindo uma representação da forma $f(x)=\sum_{n=0}^{\infty} f_{n} x^{n}, x \in \mathbb{R}$.

Em um segundo momento, a $L^{2}$-ortogonalidade da família de monômios $\left\{x^{\alpha}\right\}_{\alpha \in \mathbb{Z}_{+}^{m+1}}$ sobre a esfera complexa $([53,63])$ nos motivou a investigar também o decaimento dos autovalores dos operadores integrais $\mathcal{K}$ gerados por núcleos mensuráveis $K: X \times X \rightarrow$ $\mathbb{C}$, possuindo representação em série na forma

$$
K(x, y)=\sum_{\alpha \in \mathbb{Z}_{+}^{m+1}} a_{\alpha} f_{\alpha}(x) \overline{f_{\alpha}(y)}, \quad x, y \in X
$$

A investigação é feita no caso em que $X$ é um subconjunto não-vazio de $\mathbb{R}^{m+1}$ (ou $\mathbb{C}^{m+1}$ ) munido da medida de Lebesgue $\nu$ e $\left\{f_{\alpha}\right\}_{\alpha \in \mathbb{Z}_{+}^{m+1}}$ é um conjunto $L^{2}(X, \nu)$-ortogonal. A análise neste caso também é feita com a presença da hipótese

$$
\sum_{\alpha \in \mathbb{Z}_{+}^{m+1}}\left|a_{\alpha}\right|\left\|f_{\alpha}\right\|_{2}^{2}<\infty .
$$

O trabalho em si está organizado da seguinte forma. No Capítulo 1, introduzimos resultados básicos e clássicos necessários para a obtenção dos resultados originais que compõem este trabalho. No Capítulo 2, discutimos uma representação em série para operadores integrais que é intrínseca a uma classe de operadores a qual inclui os descritos nesta introdução. Já nos dois capítulos seguintes iniciamos a abordagem sobre decaimento dos autovalores dos operadores em questão. Fazemos esta análise em duas etapas: no Capítulo 3 consideramos o caso dos núcleos em série de potências sobre a esfera real $S^{m}$ e, posteriormente, estendemos esta análise aos núcleos em série de potências, definidos sobre a bola unitária fechada de $\mathbb{R}^{m+1}$. Em seguida, no Capítulo 4 abordamos os núcleos gerados por séries de funções $L^{2}(X, \nu)$-ortogonais acima introduzidos, os quais incluem os núcleos com representação em série de potências definidos sobre a esfera complexa. Ao final de ambos capítulos, alguns exemplos são apresentados buscando ilustrar aplicações dos resultados obtidos. O Capítulo 5 é reservado à discussão de alguns problemas interessantes que surgiram ao longo do caminho e que ainda estamos investigando ou que, acreditamos, ainda precisam ser analisados. 


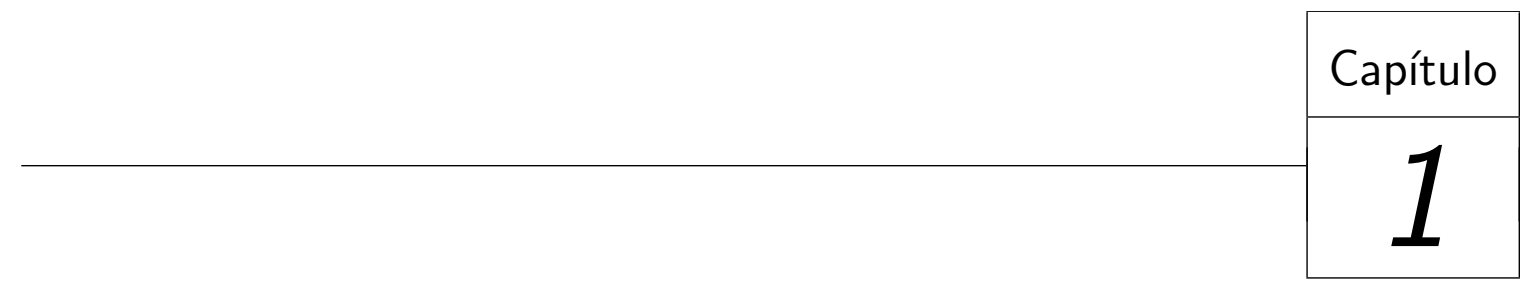

Preliminares

Neste capítulo, introduzimos alguns conceitos básicos e listamos alguns resultados técnicos necessários para o desenvolvimento da tese. Aqueles resultados que acreditamos ser novos ou de difícil localização na literatura serão devidamente justificados. Referências para os demais resultados serão citadas em pontos convenientes do capítulo. O leitor interessado na essência do trabalho, pode iniciar a leitura do mesmo pelo Capítulo 2, retornando ao Capítulo 1 em caso de necessidade.

\subsection{Resultados da análise clássica}

Alguns dos resultados principais neste trabalho descrevem a velocidade com que sequências decrescentes de números reais se aproximam de zero. Faremos uso da notação da Landau, introduzidas a seguir, para descrever o decaimento de sequências.

Se $\left\{a_{n}\right\}$ e $\left\{b_{n}\right\}$ são sequências de números reais positivos, escreveremos

$$
a_{n}=O\left(b_{n}\right), \quad(n \rightarrow \infty),
$$

para indicar que

$$
a_{n} \leq C b_{n}, \quad n=N+1, N+2, \ldots,
$$

para algum real positivo $C$ e algum inteiro positivo $N$. Em particular, se $a_{n}=O\left(b_{n}\right)$, $(n \rightarrow \infty)$, então podemos encontrar $C_{1} \geq C$ tal que

$$
a_{n} \leq C_{1} b_{n}, \quad n=1,2, \ldots
$$


Escreveremos

$$
a_{n}=o\left(b_{n}\right), \quad(n \rightarrow \infty),
$$

para indicar que

$$
\lim _{n \rightarrow \infty} \frac{a_{n}}{b_{n}}=0
$$

e

$$
a_{n} \sim b_{n}, \quad(n \rightarrow \infty),
$$

para indicar que

$$
\lim _{n \rightarrow \infty} \frac{a_{n}}{b_{n}}=1 .
$$

O primeiro resultado desta seção descreve o decaimento de uma sequência decrescente e somável de números reais positivos.

Lema 1.1.1. Seja $\left\{d_{n}\right\}$ uma sequência decrescente de números reais positivos. Se $\sum_{n=1}^{\infty} d_{n}<\infty$, então $d_{n}=o\left(n^{-1}\right),(n \rightarrow \infty)$.

O resultado acima é conhecido, mas não facilmente encontrado na literatura. Pretendemos usar uma generalização do mesmo, a qual segue de resultados demonstrados na referência [36]. A generalização tem papel decisivo na obtenção de algumas estimativas no Capítulo 4. Por esta razão, e pelo fato da justificativa em [36] envolver argumentos relativamente complexos, incluímos uma demonstração de nossa autoria.

Lema 1.1.2. Sejam $\left\{d_{n}\right\}$ uma sequência decrescente de números reais positivos e $r$ e $s$ números reais não-negativos fixados. Se $\sum_{n=1}^{\infty} n^{r} d_{n}^{s}<\infty$, então

$$
d_{n}=o\left(n^{-(r+1) / s}\right), \quad(n \rightarrow \infty) .
$$

Demonstração: Seja $\epsilon>0$. Se a sequência $\left\{n^{r} d_{n}^{s}\right\}$ é somável, então podemos selecionar $N>0$ tal que

$$
\sum_{n=k+1}^{2 k} n^{r} d_{n}^{s}<\frac{\epsilon}{2^{2 r+2}}, \quad k=N+1, N+2, \ldots
$$

Como $\left\{d_{n}\right\}$ é decrescente, segue que

$$
k^{r+1} d_{2 k}^{s}=\sum_{n=k+1}^{2 k} k^{r} d_{2 k}^{s} \leq \sum_{n=k+1}^{2 k} n^{r} d_{n}^{s}<\frac{\epsilon}{2^{2 r+2}}, \quad k=N+1, N+2, \ldots,
$$

ou seja,

$$
(2 k)^{r+1} d_{2 k}^{s}<\frac{\epsilon}{2^{r+1}}<\epsilon, \quad k=N+1, N+2, \ldots
$$


Como

$$
(2 k+1)^{r+1} d_{2 k+1}^{s}=\left(1+\frac{1}{2 k}\right)^{r+1}(2 k)^{r+1} d_{2 k+1}^{s} \leq 2^{r+1}(2 k)^{r+1} d_{2 k}^{s} .
$$

podemos concluir também que

$$
(2 k+1)^{r+1} d_{2 k+1}^{s}<\epsilon, \quad k=N+1, N+2, \ldots
$$

Portanto,

$$
k^{r+1} d_{k}^{s}<\epsilon, \quad k=N+1, N+2, \ldots,
$$

o que conclui a demonstração.

O próximo resultado é uma consequência do teste da integral para convergência de séries. Ele descreve a velocidade de convergência de uma série bem conhecida, fato a ser utilizado na obtenção de algumas estimativas no Capítulo 3. Uma justificativa detalhada pode ser encontrada em [33, p. 65].

Lema 1.1.3. Se $n$ é um inteiro positivo e r é um número real maior que 1, então

$$
\sum_{k=n}^{\infty} \frac{1}{k^{r}}=O\left(\frac{1}{n^{r-1}}\right), \quad(n \rightarrow \infty) .
$$

Em alguns casos, é possível descrever o decaimento de uma sequência a partir do decaimento de algumas de suas subsequências. O próximo resultado descreve uma situação deste tipo. Daqui em diante, escreveremos $\mathbb{N}=\{0,1,2, \ldots\}$. Se $l$ e $j$ são inteiros, escreveremos ainda $l \mathbb{N}+j=\{l+j, 2 l+j, \ldots\}$.

Lema 1.1.4. Seja $\left\{c_{n}\right\}$ uma sequência de números reais não-negativos decrescendo para 0. Fixados inteiros positivos $l$ e $m$, considere a subsequência $\left\{c_{n}^{\prime}\right\}$ de $\left\{c_{n}\right\}$ definida pela fórmula $c_{n}^{\prime}=c_{(l n)^{m}}, n=1,2, \ldots$ Se t é um número real e $c_{n}^{\prime}=O\left(n^{-t}\right),(n \rightarrow \infty)$, então $c_{n}=O\left(n^{-t / m}\right),(n \rightarrow \infty)$.

Demonstração: Seja $t$ um número real e suponha que

$$
c_{(l n)^{m}} \leq \frac{C}{n^{t}}, \quad n=1,2, \ldots
$$

Como $\left\{c_{n}\right\}$ é decrescente, a desigualdade 1.1 .4 implica na existência de $C_{1}>0$ tal que

$$
c_{(l n+j)^{m}} \leq \frac{C_{1}}{(\ln +j)^{t}}, \quad n=1,2, \ldots, \quad j=1,2, \ldots, l-1 .
$$

Como $\mathbb{N}=\cup_{j=0}^{l-1}(l \mathbb{N}+j)$, segue que

$$
c_{n^{m}} \leq \frac{C_{1}}{n^{t}}, \quad n=1,2, \ldots
$$


Usando novamente a monotonicidade de $\left\{c_{n}\right\}$, vem que

$$
c_{n^{m}+1} \leq \frac{C_{1}}{\left(n^{m}\right)^{t / m}}=\frac{C_{1}}{\left(n^{m}+1\right)^{t / m}}\left(\frac{n^{m}+1}{n^{m}}\right)^{t / m}, \quad n=1,2, \ldots
$$

Indutivamente,

$$
c_{n^{m}+j} \leq \frac{C_{1}}{\left(n^{m}+j\right)^{t / m}}\left(\frac{n^{m}+j}{n^{m}}\right)^{t / m}, \quad n=1,2, \ldots,
$$

para cada $j$ no conjunto $\left\{0,1, \ldots,(n+1)^{m}-n^{m}-1\right\}$. No entanto, como

$$
j \leq(n+1)^{m}-n^{m}-1 \leq n^{m}\left[\left(1+\frac{1}{n}\right)^{m}-1\right] \leq\left(2^{m}-1\right) n^{m},
$$

vemos que

$$
\frac{n^{m}+j}{n^{m}} \leq 2^{m}, \quad j=0,1, \ldots,(n+1)^{m}-n^{m}-1, \quad n=1,2, \ldots .
$$

Combinando esta última desigualdade com (1.1.5) deduzimos que

$$
c_{n^{m}+j} \leq \frac{C_{1} 2^{t}}{\left(n^{m}+j\right)^{t / m}}, \quad j=0,1, \ldots,(n+1)^{m}-n^{m}-1, \quad n \geq 1 .
$$

O resultado segue da igualdade $\mathbb{N}=\cup_{n=1}^{\infty} \cup_{k=0}^{(n+1)^{m}-n^{m}-1}\left\{n^{m}+k\right\}$.

O próximo resultado é uma versão do resultado conhecido como teorema da aproximação de Stirling ([2, p. 18]).

Lema 1.1.5. Se $\Gamma$ denota a função gama usual, então

$$
\lim _{\operatorname{Re} x \rightarrow \infty} \frac{\Gamma(x)}{\sqrt{2 \pi} x^{x-1 / 2} e^{-x}}=1 .
$$

Em particular,

$$
n ! \sim \sqrt{2 \pi n}\left(\frac{n}{e}\right)^{n}, \quad(n \rightarrow \infty) .
$$

A seguir, introduziremos um pouco de notação básica envolvendo multi-índices. Se $\mathbb{Z}_{+}=\mathbb{N} \cup\{0\}$, usaremos a seguinte simbologia para um elemento $\alpha=\left(\alpha_{1}, \cdots, \alpha_{m+1}\right)$ de $\mathbb{Z}_{+}^{m+1}$ :

$$
|\alpha|:=\sum_{j=1}^{m+1} \alpha_{j}
$$

e

$$
k \alpha !:=\left(k \alpha_{1}\right) ! \cdots\left(k \alpha_{m+1}\right) !, \quad k \in \mathbb{Z}_{+} .
$$

O resultado a seguir pode ser encontrado em [26, p. 65] e [4, p. 78]. Estaremos utilizando a seguinte notação:

$$
b_{k}^{m+1}:=\left(\begin{array}{c}
k+m \\
m
\end{array}\right), \quad m \in \mathbb{N}, \quad k \in \mathbb{Z}_{+} .
$$


Lema 1.1.6. Fixados um inteiro positivo $m$ e um inteiro não negativo $k$, a cardinalidade do conjunto $\left\{\alpha \in \mathbb{Z}_{+}^{m+1},|\alpha|=k\right\}$ é precisamente $b_{k}^{m+1}$.

A definição de $b_{k}^{m+1}$ e a fórmula binomial

$$
\sum_{j=0}^{k-1}\left(\begin{array}{c}
j+m \\
m
\end{array}\right)=\left(\begin{array}{c}
k+m \\
m+1
\end{array}\right), \quad k=1,2, \ldots
$$

justificam a igualdade

$$
\sum_{j=0}^{k-1} b_{j}^{m+1}=b_{k-1}^{m+2}, \quad k=1,2, \ldots .
$$

O lema abaixo descreve uma desigualdade elementar para multi-índices.

Lema 1.1.7. Sejam l e $k$ inteiros não negativos e $\alpha$ um multi-índice de $\mathbb{Z}_{+}^{m+1}$. Se $l \leq k$, então

$$
\frac{(k \alpha) !}{(l \alpha) !} \leq \frac{|k \alpha| !}{|l \alpha| !}
$$

Demonstração: $\mathrm{O}$ caso $m=1$ segue da desigualdade

$$
\left(\begin{array}{c}
l \alpha_{1}+l \alpha_{2} \\
l \alpha_{1}
\end{array}\right) \leq\left(\begin{array}{c}
k \alpha_{1}+k \alpha_{2} \\
k \alpha_{1}
\end{array}\right), \quad \alpha_{1}, \alpha_{2} \in \mathbb{Z}_{+}
$$

a qual pode ser verificada diretamente. Prosseguimos utilizando indução sobre $m$. Supondo que a desigualdade a ser provada seja verdadeira para todos os multi-índices de $\mathbb{Z}_{+}^{m}$, se $\alpha \in \mathbb{Z}^{m+1}$, então

$$
\frac{\left(k \alpha_{1}\right) ! \ldots\left(k \alpha_{m}\right) !\left(k \alpha_{m+1}\right) !}{\left(l \alpha_{1}\right) ! \ldots\left(l \alpha_{m}\right) !\left(\ell \alpha_{m+1}\right) !} \leq \frac{\left(k \alpha_{1}+\cdots+k \alpha_{m}\right) !}{\left(l \alpha_{1}+\cdots+l \alpha_{m}\right) !} \frac{\left(k \alpha_{m+1}\right) !}{\left(l \alpha_{m+1}\right) !}
$$

Combinando esta desigualdade com o passo inicial da indução, obtemos

$$
\frac{\left(k \alpha_{1}\right) ! \ldots\left(k \alpha_{m}\right) !\left(k \alpha_{m+1}\right) !}{\left(l \alpha_{1}\right) ! \ldots\left(l \alpha_{m}\right) !\left(\ell \alpha_{m+1}\right) !} \leq \frac{\left(k \alpha_{1}+\cdots+k \alpha_{m}+k \alpha_{m+1}\right) !}{\left(l \alpha_{1}+\cdots+l \alpha_{m}+l \alpha_{m+1}\right) !}
$$

Isto completa a demonstração.

Observação 1.1.8. A desigualdade obtida no lema anterior é fina, já que

$$
\max _{|\alpha|=n} \frac{(k \alpha) !}{(l \alpha) !}=\frac{(k n) !}{(l n) !} .
$$

O próximo resultado é conhecido como Teorema Multinomial. A demonstração será incluída para a conveniência do leitor. 
Teorema 1.1.9. Se $n$ é um inteiro positivo e $x=\left(x_{1}, \ldots, x_{m+1}\right)$ é um elemento de $\mathbb{R}^{m+1}$, então

$$
\left(x_{1}+\cdots+x_{m+1}\right)^{n}=\sum_{|\alpha|=n} \frac{n !}{\alpha !} x^{\alpha} .
$$

Demonstração: Fixemos $n$ e $x$ como no enunciado. Utilizaremos indução sobre a dimensão $m$. Para o caso $m=0$, ambos os lados da igualdade no enunciado são iguais a $x_{1}^{n}$. Se o resultado vale para $m \geq 1$, então segue da hipótese de indução que

$$
\begin{aligned}
& \left(x_{1}+\cdots+x_{m}+x_{m+1}\right)^{n}=\left(x_{1}+\cdots+\left(x_{m}+x_{m+1}\right)\right)^{n} \\
& =\sum_{\alpha_{1}+\cdots+\alpha_{m-1}+\beta=n}\left(\begin{array}{c}
n \\
\alpha_{1}, \ldots \alpha_{m-1}, \beta
\end{array}\right) x_{1}^{\alpha_{1}} \ldots x_{m-1}^{\alpha_{m-1}}\left(x_{m}+x_{m+1}\right)^{\beta} \text {, }
\end{aligned}
$$

onde

$$
\left(\begin{array}{c}
n \\
\alpha_{1}, \ldots \alpha_{m-1}, \beta
\end{array}\right)=\frac{n !}{\alpha_{1} ! \ldots \alpha_{m-1} ! \beta !} .
$$

Utilizando o Teorema Binomial

$$
\left(x_{m}+x_{m+1}\right)^{\beta}=\sum_{\alpha_{m}+\alpha_{m+1}=\beta}\left(\begin{array}{c}
\beta \\
\alpha_{m}, \alpha_{m+1}
\end{array}\right) x_{m}^{\alpha_{m}} x_{m+1}^{\alpha_{m+1}},
$$

combinado com a igualdade

$$
\left(\begin{array}{c}
n \\
\alpha_{1}, \ldots, \alpha_{m-1}, \beta
\end{array}\right)\left(\begin{array}{c}
\beta \\
\alpha_{m}, \alpha_{m+1}
\end{array}\right)=\left(\begin{array}{c}
n \\
\alpha_{1}, \ldots, \alpha_{m-1}, \alpha_{m}, \alpha_{m+1}
\end{array}\right),
$$

concluímos que

$$
\left(x_{1}+\cdots+x_{m+1}\right)^{n}=\sum_{\alpha_{1}+\cdots+\alpha_{m+1}=n}\left(\begin{array}{c}
n \\
\alpha_{1}, \ldots, \alpha_{m+1}
\end{array}\right) x_{1}^{\alpha_{1}} \ldots x_{m+1}^{\alpha_{m+1}} .
$$

Isso encerra a demonstração.

A seguir, como na introdução, o símbolo "." denotará o produto interno usual em $\mathbb{R}^{m+1}$.

Corolário 1.1.10. Se n é um inteiro não negativo, então

$$
(x \cdot y)^{n}=\sum_{|\alpha|=n} \frac{n !}{\alpha !} x^{\alpha} y^{\alpha}, \quad x, y \in \mathbb{R}^{m+1} .
$$

Em particular,

$$
(m+1)^{n}=\sum_{|\alpha|=n} \frac{n !}{\alpha !}
$$




\subsection{Resultados da teoria da medida}

Nesta seção, introduzimos rapidamente notação e resultados conhecidos da teoria da medida que serão utilizados à frente, concluindo-a com alguns resultados relativos a integração de monômios sobre a esfera unitária de $\mathbb{R}^{m+1}$. Toda teoria apresentada aqui pode ser encontrada em [22, 52]. Havendo necessidade, outras referências serão citadas no decorrer da seção.

Se $(X, \nu)$ é um espaço de medida e $p \in(0, \infty)$, consideraremos os espaços usuais $L^{p}(X, \nu), p=1,2$. A norma nesses espaços é então dada por

$$
\|f\|_{p}:=\left[\int_{X}|f(x)|^{p} d \nu(x)\right]^{1 / p} .
$$

De particular importância neste trabalho, é o fato de $L^{2}(X, \nu)$ ser um espaço de Hilbert. O produto interno neste espaço é dado por

$$
\langle f, g\rangle_{2}:=\int_{X} f(x) \overline{g(x)} d \nu(x), \quad f, g \in L^{2}(X, \nu) .
$$

Um importante critério para se trocar os símbolos de soma e integração é como segue $([22$, p. 55]).

Teorema 1.2.1. Seja $\left\{f_{n}\right\}$ uma sequência em $L^{1}(X, \nu)$. Se

$$
\sum_{n=1}^{\infty} \int_{X}\left|f_{n}(x)\right| d \nu(x)<\infty,
$$

então $\sum_{n=1}^{\infty} f_{n}(x)$ converge, para quase todo $x \in X$. O limite é uma função de $L^{1}(X, \nu)$ $e$

$$
\sum_{n=1}^{\infty} \int_{X} f_{n}(x) d \nu(x)=\int_{X} \sum_{n=1}^{\infty} f_{n}(x) d \nu(x) .
$$

Outro resultado que merece referência neste momento é a seguinte proposição ([22, p. 186]).

Proposição 1.2.2. Se $\nu(X)<\infty$, então $L^{2}(X, \nu)$ é um subespaço de $L^{1}(X, \nu)$ e $\|f\|_{1} \leq\|f\|_{2} \nu(X)^{1 / 2}$.

Prosseguindo, temos a clássica desigualdade de Hölder, ou seja,

$$
\|f g\|_{1} \leq\|f\|_{2}\|g\|_{2}, \quad f, g \in L^{2}(X, \nu) .
$$

Ela garante, em particular, que se $f, g \in L^{2}(X, \nu)$, então $f g \in L^{1}(X, \nu)$.

O Teorema de Fubini-Tonelli garante a iteração de integrais no espaço produto $L^{1}(X \times X, \nu \times \nu)([52$, p. 307];[22, p. 67]). 
Teorema 1.2.3. Sejam $(X, \nu)$ e $(Y, \mu)$ espaços de medida completos (ou $\sigma$-finitos) e $f$ um elemento de $L^{1}(X \times Y, \nu \times \mu)$. Então as fórmulas

$$
g(x)=\int_{Y} f(x, y) d \mu(y), \quad x \in X \text { q.s }
$$

$e$

$$
h(y)=\int_{X} f(x, y) d \nu(x), \quad y \in Y \text { q.s. }
$$

definem elementos de $L^{1}(X, \nu)$ e $L^{1}(Y, \mu)$ respectivamente. Além disso, vale a fórmula

$$
\int_{X \times Y} f d(\nu \times \mu)=\int_{X}\left[\int_{Y} f(x, y) d \mu(y)\right] d \nu(x)=\int_{Y}\left[\int_{X} f(x, y) d \nu(x)\right] d \mu(y) .
$$

Completaremos a seção com três resultados técnicos envolvendo a integração de monômios, no caso em que $X$ é a esfera unitária $S^{m}$ de $\mathbb{R}^{m+1}$. Como usualmente feito na literatura, a medida usual sobre $S^{m}$ será denotada por $\sigma_{m}$. Os três resultados entram de maneira decisiva nas demonstrações de alguns dos resultados principais da tese a serem apresentados no Capítulo 3. Considerando a notação multi-indexada introduzida na seção anterior e fixado um multi-índice $\alpha$ de $\mathbb{Z}_{+}^{m+1}$, a fórmula abaixo define o monômio determinado por $\alpha$ sobre $S^{m}$ :

$$
x^{\alpha}:=x_{1}^{\alpha_{1}} \ldots x_{m+1}^{\alpha_{m+1}}, \quad x=\left(x_{1}, \ldots, x_{m+1}\right) \in S^{m}, \quad \alpha=\left(\alpha_{1}, \ldots, \alpha_{m+1}\right) \in \mathbb{Z}_{+}^{m+1} .
$$

Ainda com relação a definição acima, o número $|\alpha|$ é chamado de grau do monômio.

No lema abaixo, apresentamos uma fórmula para computar a integral de um monômio sobre $S^{m}$. Nossa prova é baseada em argumentos oriundos de [8] e [22].

Lema 1.2.4. Se $\alpha$ é um multi-índice em $\mathbb{Z}_{+}^{m+1}$, então

$$
\int_{S^{m}} x^{\alpha} d \sigma_{m}(x)= \begin{cases}\frac{2 \prod_{j=1}^{m+1} \Gamma\left(\left(\alpha_{j}+1\right) / 2\right)}{\Gamma((|\alpha|+m+1) / 2)}, & \text { se cada } \alpha_{j} \text { é par } \\ 0, & \text { caso contrário. }\end{cases}
$$

Demonstração: Fixe um multi-índice $\alpha \in \mathbb{Z}_{+}^{m+1}$. Se $\alpha=\left(\alpha_{1}, \ldots, \alpha_{j}, \ldots, \alpha_{m+1}\right)$ é tal que $\alpha_{j}$ é ímpar, então a invariância de $d \sigma_{m}$ por transformações ortogonais de $\mathbb{R}^{m+1}$ implica que

$$
\begin{aligned}
\int_{S^{m}} x^{\alpha} d \sigma_{m}(x) & =-\int_{S^{m}} x_{1}^{\alpha_{1}} \ldots\left(-x_{j}\right)^{\alpha_{j}} \ldots x_{m+1}^{\alpha_{m+1}} d \sigma_{m}(x) \\
& =-\int_{S^{m}} x^{\alpha} d \sigma_{m}(x) .
\end{aligned}
$$

Suponhamos então que cada $\alpha_{j}$ é par e computemos a integral

$$
J_{1}:=\int_{\mathbb{R}^{m+1}} y^{\alpha} e^{-|y|^{2}} d y
$$


via coordenadas polares ([22, p. 78]):

$$
\begin{aligned}
J_{1} & =\int_{0}^{\infty} \int_{S^{m}}(t x)^{\alpha} t x e^{-t^{2}} d \sigma_{m}(x) d t \\
& =\int_{0}^{\infty} t^{|\alpha|+m} e^{-t^{2}} d t \int_{S^{m}} x^{\alpha} d \sigma_{m}(x) .
\end{aligned}
$$

Utilizando a definição da função gama na última desigualdade acima, ou seja, a fórmula

$$
\Gamma(u)=\int_{0}^{\infty} t^{u-1} e^{-t} d t, \quad \operatorname{Re}(u)>0,
$$

obtemos

$$
J_{1}=\frac{1}{2} \Gamma((|\alpha|+m+1) / 2) \int_{S^{m}} x^{\alpha} d \sigma_{m}(x) .
$$

No entanto, computação direta da mesma integral, nos leva a

$$
J_{1}=\prod_{i=1}^{m+1} \int_{-\infty}^{\infty} y_{i}^{\alpha_{i}} e^{-y_{i}^{2}} d y_{i}=2 \prod_{i=1}^{m+1} \int_{0}^{\infty} y_{i}^{\alpha_{i}} e^{-y_{i}^{2}} d y_{i} .
$$

Introduzindo a função gama novamente, chegamos a

$$
J_{1}=\prod_{i=1}^{m+1} \Gamma\left(\left(\alpha_{i}+1\right) / 2\right) .
$$

Comparando as identidades obtidas concluímos a demonstração.

Uma consequência imediata do lema é como segue.

Proposição 1.2.5. Se $\alpha$ é um multi-índice em $\mathbb{Z}_{+}^{m+1}$, então

$$
\int_{S^{m}} x^{2 \alpha} d \sigma_{m}(x)=\frac{2(2 \alpha) ! \pi^{(m+1) / 2}}{4^{|\alpha|} \alpha ! \Gamma(|\alpha|+(m+1) / 2)} .
$$

Demonstração: Fixe $\alpha \in \mathbb{Z}_{+}^{m+1}$. Aplicando o lema anterior deduzimos que

$$
\int_{S^{m}} x^{2 \alpha} d \sigma_{m}(x)=\frac{2 \Gamma\left(\alpha_{1}+1 / 2\right) \ldots \Gamma\left(\alpha_{m+1}+1 / 2\right)}{\Gamma(|\alpha|+(m+1) / 2)} .
$$

Uma aplicação da fórmula elementar ([1, p. 255])

$$
\Gamma(n+1 / 2)=\frac{(2 n) ! \sqrt{\pi}}{4^{n} n !}, \quad n=1,2, \ldots,
$$

produz a fórmula no enunciado da proposição.

Para o último resultado desta seção, é conveniente agregar a seguinte notação:

$$
p_{\alpha}(x)=x^{\alpha}, \quad x \in S^{m}, \quad \alpha \in \mathbb{Z}_{+}^{m+1} .
$$

A partir da proposição anterior, a qual apresenta uma fórmula explícita para o cálculo de $\left\|p_{\alpha}\right\|_{2}$, o resultado a seguir descreve o comportamento assintótico da sequência $\left\{\left\|p_{\alpha}\right\|_{2}\right\}$, quando $|\alpha| \rightarrow \infty$. 
Teorema 1.2.6. A sequência $\left\{\left\|p_{\alpha}\right\|_{2}\right\}$ satisfaz

$$
\left\|p_{\alpha}\right\|_{2}^{2}=O\left(|\alpha|^{-m / 2}\right), \quad(|\alpha| \rightarrow \infty)
$$

Demonstração: Utilizando ambos, a Proposição 1.2 .5 e o Lema 1.1 .7 com $k=2$ e $l=1$, obtemos

$$
\left\|p_{\alpha}\right\|_{2}^{2}=\frac{2(2 \alpha) ! \pi^{(m+1) / 2}}{4^{|\alpha|} \alpha ! \Gamma(|\alpha|+(m+1) / 2)} \leq \frac{2|2 \alpha| ! \pi^{(m+1) / 2}}{4^{|\alpha|}|\alpha| ! \Gamma(|\alpha|+(m+1) / 2)}, \quad \alpha \in \mathbb{Z}_{+}^{m+1} .
$$

Como o teorema da aproximação de Stirling nos dá que

$$
\frac{(2 n) !}{n ! \Gamma(n+(m+1) / 2)} \sim \frac{e^{(m+1) / 2}}{\sqrt{2 \pi}} \frac{(2 n)^{2 n+1 / 2}}{n^{n+1 / 2}(n+(m+1) / 2)^{n+m / 2}}, \quad(n \rightarrow \infty),
$$

poucos cálculos adicionais nos levam à seguinte fórmula assintótica

$$
\frac{|2 \alpha| !}{4^{|\alpha|}|\alpha| ! \Gamma(|\alpha|+(m+1) / 2)} \sim \frac{\pi^{-1 / 2}}{|\alpha|^{m / 2}}, \quad(|\alpha| \rightarrow \infty) .
$$

Isto conclui a demonstração.

\subsection{Resultados da análise funcional}

Nesta seção incluímos alguns resultados básicos sobre operadores compactos em espaços de Hilbert e ainda um pouco da análise espectral envolvendo tais operadores. Alguns dos resultados já estão formatados visando sua utilização no texto. Todos estes resultados bem como outros adicionais podem ser encontrados em [21, 25, 60].

Se $\mathcal{X}$ e $\mathcal{Y}$ são espaços vetoriais normados, escreveremos $\mathcal{L}(\mathcal{X}, \mathcal{Y})$ para indicar o espaço vetorial de todas transformações lineares de $\mathcal{X}$ em $\mathcal{Y}$ que são limitadas. Quando $\mathcal{X}=\mathcal{Y}$, escreveremos $\mathcal{L}(\mathcal{X}, \mathcal{Y})=\mathcal{L}(\mathcal{X})$ e seus elementos serão chamados de operadores sobre $\mathcal{X}$.

Definição 1.3.1. Sejam $\mathcal{X}$ e $\mathcal{Y}$ espaços de Banach. Um elemento $T$ de $\mathcal{L}(\mathcal{X}, \mathcal{Y})$ é compacto quando a imagem de cada sequência limitada de $\mathcal{X}$ possui uma subsequência convergente em $\mathcal{Y}$.

Um exemplo elementar de transformação linear compacta é fornecido pelo teorema abaixo.

Teorema 1.3.2. Sejam $\mathcal{X}$ e $\mathcal{Y}$ espaços de Banach. Se $\left\{T_{n}\right\}$ é uma sequência em $\mathcal{L}(\mathcal{X}, \mathcal{Y})$, cada $T_{n}$ tem posto finito e $\left\{T_{n}\right\}$ converge em $\mathcal{L}(\mathcal{X}, \mathcal{Y})$, então o limite é um elemento compacto de $\mathcal{L}(\mathcal{X}, \mathcal{Y})$. 
No contexto de espaços de Hilbert, o teorema anterior pode ser refinado. No restante da seção, usaremos o símbolo $\mathcal{H}$ para denotar um espaço de Hilbert arbitrário. Se necessário, seu produto interno será denotado por $\langle\cdot, \cdot\rangle_{\mathcal{H}}$. Note que, $\mathcal{L}(\mathcal{H})$ é um espaço de Banach.

Teorema 1.3.3. Seja $T$ um elemento de $\mathcal{L}(\mathcal{H})$. Então $T$ é um operador compacto se, $e$ somente se, seu adjunto $T^{*}$ é compacto. Ainda, cada operador compacto de $\mathcal{L}(\mathcal{H})$ pode ser aproximado, na norma deste espaço, por uma sequência $\left\{T_{n}\right\}$ de $\mathcal{L}(\mathcal{H})$, inteiramente formada por operadores de posto finito.

A definição abaixo contempla outra classe de operadores lineares sobre um espaço de Hilbert de importância neste trabalho.

Definição 1.3.4. Um operador $T$ em $\mathcal{L}(\mathcal{H})$ é positivo quando

$$
\langle T(v), v\rangle_{\mathcal{H}} \geq 0, \quad v \in \mathcal{H} .
$$

Se $T \in \mathcal{L}(\mathcal{H})$, então $T^{*} T$ é automaticamente autoadjunto e também positivo, uma vez que

$$
\left\langle T^{*} T(v), v\right\rangle_{\mathcal{H}}=\langle T(v), T(v)\rangle_{\mathcal{H}}=\|T(v)\|_{\mathcal{H}}^{2} \geq 0, \quad v \in \mathcal{H} .
$$

A identidade de polarização pode ser usada para ratificar o seguinte resultado ([60, p. 72]).

Teorema 1.3.5. Seja $\mathcal{H}$ um espaço de Hilbert complexo. Se um elemento $T$ em $\mathcal{L}(\mathcal{H})$ é um operador positivo, então T é um operador autoadjunto.

O teorema a seguir é conhecido como teorema espectral para operadores compactos e auto-adjuntos.

Teorema 1.3.6. [Hilbert-Schmidt] Seja $T$ um operador compacto em $\mathcal{L}(\mathcal{H})$. Se $T$ é auto-adjunto, então existe uma sequência ortonormal $\left\{v_{n}\right\}$ de $\mathcal{H}$ e outra sequência de números reais $\left\{\lambda_{n}(T)\right\}$ de modo que

$$
T(v)=\sum_{n=1}^{\infty} \lambda_{n}(T)\left\langle v, v_{n}\right\rangle_{\mathcal{H}} v_{n}, \quad v \in \mathcal{H},
$$

$\operatorname{com}\left|\lambda_{1}(T)\right| \geq\left|\lambda_{2}(T)\right| \geq \cdots \geq 0$ e $\lim _{n \rightarrow \infty} \lambda_{n}(T)=0$.

Corolário 1.3.7. Nas condições do Teorema de Hilbert-Schmidt, valem as seguintes conclusões adicionais:

(i) Se T é positivo, então $\lambda_{n}(T) \geq 0, n=1,2, \ldots$;

(ii) Se $\mathcal{H}$ é separável, então podemos supor que $\left\{v_{n}\right\}$ é uma base ortonormal de $\mathcal{H}$. 
Demonstração: Para provar $(i)$ basta notar que $T\left(v_{n}\right)=\lambda_{n} v_{n}, n=1,2, \ldots$ e que quando $T(v)=\lambda v$ e $v \neq 0$ então $0 \leq\langle T(v), v\rangle_{\mathcal{H}}=\lambda\langle v, v\rangle_{\mathcal{H}}$. O item (ii) segue da equivalência entre a separabilidade de $\mathcal{H}$ e a existência de uma base ortonormal enumerável para $\mathcal{H}$.

Antes de prosseguir, se $T$ é um elemento de $\mathcal{L}(\mathcal{H})$, definamos $|T|:=\left(T^{*} T\right)^{1 / 2}$. Observe que $|T|=T$ quando $T$ é autoadjunto e positivo.

Se $T$ é um operador compacto sobre um espaço de Hilbert $\mathcal{H}$, então $|T|$ é autoadjunto, compacto e positivo. Logo, o Teorema de Hilbert-Schmidt é aplicável para este operador.

Definição 1.3.8. Seja $T$ um operador compacto em $\mathcal{L}(\mathcal{H})$. Um valor singular de $T$ é um autovalor de $|T|$.

Retornando à notação do Teorema de Hilbert-Schmidt agora aplicado a $|T|$ e respeitando a ordenação dos autovalores por ele imposta, temos que

$$
\lambda_{1}(|T|) \geq \lambda_{2}(|T|) \geq \cdots \geq 0,
$$

levando-se em conta possíveis repetições relacionadas com a multiplicidade algébrica de cada um deles. Em geral, escrevemos

$$
s_{n}(T):=\lambda_{n}(|T|), \quad n=1,2, \ldots
$$

Na análise do decaimento dos autovalores de operadores compactos e auto-adjuntos, alguns resultados mais finos de análise espectral são necessários. Dois deles são descritos abaixo $([25,34])$.

Teorema 1.3.9. Se $T$ é um operador compacto e auto-adjunto em $\mathcal{L}(\mathcal{H})$, então

$$
s_{n}(T)=\left|\lambda_{n}(T)\right|, \quad n=1,2, \ldots
$$

Particularmente, se $T$ é positivo, então $s_{n}(T)=\lambda_{n}(T), n=1,2, \ldots$

No próximo resultado, $\theta(S)$ indicará o posto de um operador $S$ em $\mathcal{L}(\mathcal{H})$ e $\|\cdot\|$ a norma de um operador em $\mathcal{L}(\mathcal{H})$.

Teorema 1.3.10. Sejam $T$ um operador compacto e auto-adjunto em $\mathcal{L}(\mathcal{H})$. Então

$$
s_{n+1}(T)=\min \{\|T-S\|: S \in \mathcal{L}(\mathcal{H}) ; \theta(S) \leq n\}, \quad n=1,2, \ldots
$$

Consideraremos a partir de agora um tipo especial de operador linear, aquele presente no título dessa tese. Utilizamos aqui algumas notações da seção anterior. 
Definição 1.3.11. Seja $T$ um elemento de $\mathcal{L}\left(L^{2}(X, \nu)\right)$. Diremos que $T$ é um operador integral sobre $L^{2}(X, \nu)$ se existir uma função $K: X \times X \rightarrow \mathbb{C}$ tal que

$$
T(f)(x)=\int_{X} K(x, y) f(y) d \nu(y), \quad f \in L^{2}(X, \nu), \quad x \in X \text { q.s.. }
$$

Quando este for o caso, escrevemos $T=\mathcal{K}$ e diremos que $\mathcal{K}$ é o operador integral gerado pelo núcleo $K$. Alternativamente, diremos ainda que $K$ é o núcleo gerador de $\mathcal{K}$ ou que $K$ gera o operador $\mathcal{K}$ ou, simplesmente, $K$ é um núcleo, deixando implícito o operador $\mathcal{K}$.

Se o núcleo gerador $K$ do operador integral $\mathcal{K}$ é um elemento de $L^{2}(X \times X, \nu \times \nu)$, o Teorema de Fubini-Tonelli e a desigualdade de Hölder garantem que

$$
\begin{aligned}
\|\mathcal{K}(f)\|_{2}^{2} & =\int_{X}|\mathcal{K}(f)(x)|^{2} d \nu(x) \\
& =\int_{X}\left|\int_{X} K(x, y) f(y) d \nu(y)\right|^{2} d \nu(x) \\
& \leq \int_{X}\left(\left(\int_{X}|K(x, y)|^{2} d \nu(y)\right)^{1 / 2}\left(\int_{X}|f(y)|^{2} d \nu(y)\right)^{1 / 2}\right)^{2} d \nu(x) \\
& =\|K\|_{2}^{2}\|f\|_{2}^{2}, \quad f \in L^{2}(X, \nu),
\end{aligned}
$$

ou seja, $\|\mathcal{K}\| \leq\|K\|_{2}$.

O lema a seguir descreve uma propriedade um pouco mais refinada dos operadores integrais.

Lema 1.3.12. Seja $\mathcal{K}$ um operador integral sobre $L^{2}(X, \nu)$ com núcleo gerador $K$ em $L^{2}(X \times X, \nu \times \nu)$. Então $\mathcal{K}$ é compacto e seu adjunto é dado por

$$
\mathcal{K}^{*}(f)(x)=\int_{X} \overline{K(x, y)} f(y) d \nu(y), \quad x \in X .
$$

Em particular, se $K$ é hermiteano, ou seja, $K(x, y)=\overline{K(y, x)}, x, y \in X$, então $\mathcal{K}=\mathcal{K}^{*}$.

O seguinte resultado adicional sobre valores singulares de operadores integrais está demonstrado em [34, p. 9].

Teorema 1.3.13. Se o núcleo gerador $K$ de um operador integral $\mathcal{K}$ é um elemento de $L^{2}(X \times X, \nu \times \nu)$, então

$$
\sum_{n=1}^{\infty} s_{n}^{2}(\mathcal{K})=\|K\|_{2}^{2} .
$$

Combinando o Lema 1.1.1 com o teorema anterior, temos o seguinte comportamento assintótico.

Corolário 1.3.14. Se o núcleo gerador $K$ de um operador integral $\mathcal{K}$ é um elemento de $L^{2}(X \times X, \nu \times \nu)$, então $s_{n}(\mathcal{K})=o\left(n^{-1 / 2}\right),(n \rightarrow \infty)$. 

Capítulo

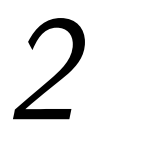

\section{Operadores integrais gerados por séries de potências.}

Neste capítulo, indicamos por $X$ um subconjunto não vazio de $\mathbb{R}^{m+1}$ ou $\mathbb{C}^{m+1}$, munido da medida de Lebesgue $\mu$ induzida e abreviamos por $\sum_{\alpha}$ as somas indexadas em $\mathbb{Z}_{+}^{m+1}$.

A idéia aqui é considerar núcleos mensuráveis da forma

$$
K(z, w)=\sum_{|\alpha|=0}^{\infty} a_{\alpha} f_{\alpha}(z) \overline{f_{\alpha}(w)}, \quad x, y \in X
$$

onde cada $a_{\alpha}$ é um número complexo, cada $f_{\alpha}$ é uma função de $L^{2}(X, \mu)$ e investigar algumas propriedades do operador $\mathcal{K}$ necessárias para a obtenção dos resultados principais deste trabalho. Em geral, uma hipótese minimal que a sequência $\left\{f_{\alpha}: \alpha \in \mathbb{Z}_{+}^{m+1}\right\}$ deveria satisfazer é ser linearmente independente. Implicitamente, isto será feito, já que nos resultados principais, teremos a hipótese de ortogonalidade da sequência.

Lembramos ao leitor que os núcleos em séries de potências, ou seja, os núcleos da forma

$$
K(x, y)=\sum_{|\alpha|=0}^{\infty} a_{\alpha} x^{\alpha} \bar{y}^{\alpha}, \quad x, y \in X,
$$

onde cada $a_{\alpha}$ é um número complexo, se encaixam na descrição acima.

Neste ponto enfatizamos que é conveniente fixar uma ordem parcial $\preceq$ em $\mathbb{Z}_{+}^{m+1}$, satisfazendo $\alpha \preceq \beta$ sempre que $|\alpha| \leq|\beta|$. Em particular, todas as somas e convergências estarão supostamente atreladas a esta ordem, a menos de notação ou menção 
em contrário. Este adicional, apesar de não ser imprescindível, facilita o entendimento dos resultados e está plenamente de acordo com todos os exemplos concretos que serão explorados ao longo do trabalho. Utilizaremos a seguinte notação para sequências multiindexadas: $\left\{a_{\alpha}\right\}:=\left\{a_{\alpha}: \alpha \in \mathbb{Z}_{+}^{m+1}\right\}$.

\subsection{Positividade de $\mathcal{K}$}

O objetivo principal nesta seção é estabelecer uma condição simples que garanta a positividade do operador integral $\mathcal{K}$ gerado por 2.0.1.

Um núcleo $K$ como descrito em 2.0.1 é diagonalmente absolutamente integrável quando a sequência $\left\{a_{\alpha}\right\}$ satisfaz

$$
\sum_{\alpha}\left|a_{\alpha}\right|\left\|f_{\alpha}\right\|_{2}^{2}<\infty
$$

Neste caso, escreveremos $K \in D A I(X)$.

Na primeira proposição abaixo, deduziremos que o operador $\mathcal{K}$ está bem posto no sentido de $L^{2}(X, \mu)$, além de ser compacto.

Proposição 2.1.1. Seja $K$ como em 2.0.1). Se $K$ é um elemento de $D A I(X)$, então $\mathcal{K}$ é um operador compacto sobre $L^{2}(X, \mu)$.

Demonstração: Suponha que $\sum_{\alpha}\left|a_{\alpha}\right|\left\|f_{\alpha}\right\|_{2}^{2}<\infty$. Utilizando o Teorema de FubiniTonelli e o Teorema 1.2.1 vemos que

$$
\int_{X} \sum_{\alpha}\left|a_{\alpha}\right|\left|f_{\alpha}(z)\right|^{2} d \mu(z) \int_{X} \sum_{\alpha}\left|a_{\alpha}\right|\left|f_{\alpha}(w)\right|^{2} d \mu(w)=\left(\sum_{\alpha}\left|a_{\alpha}\right|\left\|f_{\alpha}\right\|_{2}^{2}\right)^{2} .
$$

Combinando esta última desigualdade com a desigualdade de Hölder obtemos

$$
\int_{X} \int_{X}\left(\sum_{\alpha}\left|a_{\alpha}\right|^{1 / 2}\left|f_{\alpha}(z)\right|\left|a_{\alpha}\right|^{1 / 2}\left|f_{\alpha}(w)\right|\right)^{2} d \mu(z) d \mu(w) \leq\left(\sum_{\alpha}\left|a_{\alpha}\right|\left\|f_{\alpha}\right\|_{2}^{2}\right)^{2} .
$$

Por outro lado, segue do teorema de Fubini-Tonelli que

$$
\begin{aligned}
\|K\|_{2}^{2} & =\int_{X \times X}\left|\sum_{\alpha} a_{\alpha} f_{\alpha}(z) \overline{f_{\alpha}(w)}\right|^{2} d(\mu \times \mu)(z, w) \\
& \leq \int_{X} \int_{X}\left(\sum_{\alpha}\left|a_{\alpha}\right|^{1 / 2}\left|f_{\alpha}(z)\right|\left|a_{\alpha}\right|^{1 / 2}\left|f_{\alpha}(w)\right|\right)^{2} d \mu(z) d \mu(w) .
\end{aligned}
$$

Assim, $\|K\|_{2}^{2}<\infty$ e a conclusão da proposição segue do Lema 1.3 .12 e de 1.3 .1 . 
Observação 2.1.2. Se cada elemento da sequência $\left\{a_{\alpha}\right\}$ é um número real, então

$$
K(x, y)=\overline{K(y, x)}=K(y, x), \quad x, y \in X
$$

e, consequentemente, $\mathcal{K}$ é auto-adjunto. De fato, isso segue da fórmula

$$
\mathcal{K}^{*}(f)(x)=\int_{X} \overline{K(y, x)} f(y) d \mu(y), \quad x \in X, \quad f \in L^{2}(X, \mu) .
$$

No próximo resultado, suporemos que cada coeficiente $a_{\alpha}$ é positivo. Neste caso, a hipótese $K \in D A I(X)$ corresponde a $K$ ser diagonalmente integrável, ou seja, ao fato da aplicação $\kappa: X \rightarrow[0, \infty)$ dada por

$$
\kappa(z):=K(z, z)=\sum_{\alpha} a_{\alpha}\left|f_{\alpha}(z)\right|^{2}, \quad z \in X
$$

ser um elemento de $L^{1}(X, \mu)$.

Proposição 2.1.3. Seja $K$ como em 2.0.1). Se cada $a_{\alpha}$ é não-negativo e $\kappa$ é um elemento de $L^{1}(X, \mu)$, então $\mathcal{K}: L^{2}(X, \mu) \rightarrow L^{2}(X, \mu)$ é positivo.

Demonstração: Como $a_{\alpha} \geq 0, \alpha \in \mathbb{Z}_{+}^{m+1}$, o Teorema de Fubini-Tonelli e a desigualdade de Hölder justificam a desigualdade

$$
\sum_{\alpha} a_{\alpha} \int_{X \times X}\left|f_{\alpha}(z) \overline{f_{\alpha}(w)} f(z) \overline{f(w)}\right| d(\mu \times \mu)(z, w) \leq\|f\|_{2}^{2} \sum_{\alpha} a_{\alpha}\left\|f_{\alpha}\right\|_{2}^{2}, \quad f \in L^{2}(X, \mu) .
$$

Como Teorema 1.2.1 permite, neste caso, a comutação entre integral e a soma, deduzimos que

$$
\langle\mathcal{K}(f), f\rangle_{2}=\sum_{\alpha} a_{\alpha} \int_{X \times X} \overline{f_{\alpha}(z)} f_{\alpha}(w) f(z) \overline{f(w)} d(\mu \times \mu)(z, w), \quad f \in L^{2}(X, \mu) .
$$

Uma outra utilização do Teorema de Fubini-Tonelli nos leva a

$$
\langle\mathcal{K}(f), f\rangle_{2}=\sum_{\alpha} a_{\alpha} \int_{X} \overline{f_{\alpha}(z)} f(z) d \mu(z) \int_{X} f_{\alpha}(w) \overline{f(w)} d \mu(w), \quad f \in L^{2}(X, \mu),
$$

ou seja,

$$
\langle\mathcal{K}(f), f\rangle_{2}=\sum_{\alpha} a_{\alpha}\left|\left\langle f, f_{\alpha}\right\rangle_{2}\right|^{2} \geq 0, \quad f \in L^{2}(X, \mu) .
$$

A prova está completa. 


\subsection{Representação em série para o operador integral}

Ainda mantendo um núcleo $K$ da forma (2.0.1), o objetivo nesta seção é estabelecer condições para que o operador integral $\mathcal{K}$ tenha um representação em série. Essencialmente, se $K$ é oriundo da classe $D A I(X)$, não é necessário agregar hipóteses adicionais significativas para se obter alguma representação em série para $\mathcal{K}$.

No primeiro resultado abaixo, obtemos uma representação em série que é absolutamente convergente.

Proposição 2.2.1. Seja K como em 2.0.1). Suponha que:

(i) $\sup _{\alpha}\left|f_{\alpha}(z)\right|<\infty, z \in X$;

(ii) $\sum_{\alpha}\left|a_{\alpha}\right|\left\|f_{\alpha}\right\|_{2}<\infty$.

Então $\mathcal{K}$ possui uma representação em série na forma

$$
\mathcal{K}(f)(z)=\sum_{\alpha} a_{\alpha}\left\langle f, f_{\alpha}\right\rangle_{2} f_{\alpha}(z), \quad z \in X, \quad f \in L^{2}(X, \mu)
$$

Demonstração: Primeiramente, note que uma aplicação da desigualdade de CauchySchwarz nos leva a

$$
\sum_{\alpha}\left|a_{\alpha}\right|\left|\left\langle f, f_{\alpha}\right\rangle_{2}\right|\left|f_{\alpha}(z)\right| \leq\|f\|_{2} \sup _{\alpha}\left|f_{\alpha}(z)\right| \sum_{\alpha}\left|a_{\alpha}\right|\left\|f_{\alpha}\right\|_{2}<\infty, \quad z \in X .
$$

Aplicando o Teorema 1.2.1 para comutar a integral e a soma na expressão que define $\mathcal{K}$, obtemos

$$
\begin{aligned}
\mathcal{K}(f)(z) & =\int_{X} \sum_{\alpha} a_{\alpha} f_{\alpha}(z) \overline{f_{\alpha}(w)} f(w) d \mu(w) \\
& =\sum_{\alpha} a_{\alpha}\left\langle f, f_{\alpha}\right\rangle_{2} f_{\alpha}(z), \quad z \in X, \quad f \in L^{2}(X, \mu) .
\end{aligned}
$$

Isso finaliza a demonstração.

Os resultados a seguir descrevem caminhos para que, ao final, a convergência da série do resultado anterior também ocorra em $L^{2}(X, \mu)$.

Proposição 2.2.2. Seja $K$ como em (2.0.1). Se $K$ é um elemento de $D A I(X)$, então $\mathcal{K}$ possui uma representação em série na forma

$$
\mathcal{K}(f)=\sum_{|\alpha|=0}^{\infty} a_{\alpha}\left\langle f, f_{\alpha}\right\rangle_{2} f_{\alpha}, \quad f \in L^{2}(X, \mu),
$$

com convergência da série na norma de $L^{2}(X, \mu)$. 
Demonstração: Para cada inteiro positivo $n$, a fórmula

$$
\mathcal{K}_{n}(f)(x)=\int_{X}\left(\sum_{|\alpha|=0}^{n-1} a_{\alpha} f_{\alpha}(x) \overline{f_{\alpha}(y)}\right) f(y) d \mu(y), \quad x \in X, \quad f \in L^{2}(X, \mu),
$$

define um operador integral compacto $\mathcal{K}_{n}$ sobre $L^{2}(X, \mu)$. Como

$$
\mathcal{K}(f)(x)-\mathcal{K}_{n}(f)(x)=\int_{X}\left(\sum_{|\alpha| \geq n} a_{\alpha} f_{\alpha}(x) \overline{f_{\alpha}(y)}\right) f(y) d \mu(y), \quad x \in X, \quad f \in L^{2}(X, \mu),
$$

segue que

$$
\begin{aligned}
\left\|\mathcal{K}(f)-\mathcal{K}_{n}(f)\right\|_{2}^{2} & =\int_{X}\left[\int_{X}\left(\sum_{|\alpha| \geq n} a_{\alpha} f_{\alpha}(x) \overline{f_{\alpha}(y)}\right) f(y) d \mu(y)\right]^{2} d \mu(x) \\
& \leq \int_{X}\left[\int_{X} \sum_{|\alpha| \geq n}\left|a_{\alpha}\right|\left|f_{\alpha}(x)\right|\left|f_{\alpha}(y)\right||f(y)| d \mu(y)\right]^{2} d \mu(x),
\end{aligned}
$$

contanto que $f \in L^{2}(X, \mu)$. Se $D$ é a expressão no lado direito da desigualdade acima, invocando uma vez mais o Teorema de Fubini-Tonelli e a desigualdade de Hölder, vem que

$$
D \leq\|f\|_{2}^{2} \int_{X}\left[\sum_{|\alpha| \geq n}\left|a_{\alpha}\right|\left|f_{\alpha}(x)\right|\left\|f_{\alpha}\right\|_{2}\right]^{2} d \mu(x), \quad f \in L^{2}(X, \mu) .
$$

Novamente, a desigualdade de Hölder nos garante que

$$
\sum_{|\alpha| \geq n}\left|a_{\alpha}\right|\left|f_{\alpha}(x)\right||| f_{\alpha} \|_{2} \leq\left(\sum_{|\alpha| \geq n}\left|a_{\alpha}\right|\left|f_{\alpha}(x)\right|^{2}\right)^{1 / 2}\left(\sum_{|\alpha| \geq n}\left|a_{\alpha}\right|\left\|f_{\alpha}\right\|_{2}^{2}\right)^{1 / 2}
$$

e, por conseguinte,

$$
\left\|\mathcal{K}(f)-\mathcal{K}_{n}(f)\right\|_{2}^{2} \leq\|f\|_{2}^{2}\left(\sum_{|\alpha|=n}^{\infty}\left|a_{\alpha}\right|\left\|f_{\alpha}\right\|_{2}^{2}\right)^{2}, \quad f \in L^{2}(X, \mu) .
$$

Se $K \in D A I(X)$, é evidente que

$$
\lim _{n \rightarrow \infty}\left\|\mathcal{K}(f)-\mathcal{K}_{n}(f)\right\|_{2}=0, \quad f \in L^{2}(X, \mu) .
$$

Por outro lado, da fórmula

$$
\mathcal{K}_{n}(f)(x)=\sum_{|\alpha|=0}^{n-1} a_{\alpha}\left\langle f, f_{\alpha}\right\rangle_{2} f_{\alpha}(x), \quad x \in X, \quad f \in L^{2}(X, \mu),
$$


também temos que

$$
\lim _{n \rightarrow \infty}\left\|\sum_{|\alpha|=0}^{\infty} a_{\alpha}\left\langle f, f_{\alpha}\right\rangle_{2} f_{\alpha}-\mathcal{K}_{n}(f)\right\|_{2}=0, \quad f \in L^{2}(X, \mu) .
$$

Juntando-se as convergências obtidas, segue que

$$
\lim _{n \rightarrow \infty}\left\|\sum_{|\alpha|=0}^{\infty} a_{\alpha}\left\langle f, f_{\alpha}\right\rangle_{2} f_{\alpha}-\mathcal{K}(f)\right\|_{2}=0, \quad f \in L^{2}(X, \mu) .
$$

Isto conclui a demonstração.

O corolário a seguir elimina a possível discrepância existente entre as representações fornecidas pelos resultados acima. Em particular, ele revela que podemos ser consideravelmente tolerantes com relação à ordem parcial previamente fixada em $\mathbb{Z}_{+}^{m+1}$.

Corolário 2.2.3. Seja $K$ como em (2.0.1). Se $K$ é um elemento de $D A I(X)$, então vale a representação em série para $\mathcal{K}$

$$
\mathcal{K}(f)=\sum_{\alpha} a_{\alpha}\left\langle f, f_{\alpha}\right\rangle_{2} f_{\alpha}, \quad f \in L^{2}(X, \mu),
$$

com convergência da série na norma de $L^{2}(X, \mu)$.

Demonstração: Basta notar a seguinte desigualdade, uma consequência da desigualdade de Cauchy-Schwarz,

$$
\sum_{\alpha}\left\|a_{\alpha}\left\langle f, f_{\alpha}\right\rangle_{2} f_{\alpha}\right\|_{2} \leq\|f\|_{2} \sum_{\alpha}\left|a_{\alpha}\right|\left\|f_{\alpha}\right\|_{2}^{2}, \quad f \in L^{2}(X, \mu)
$$

Se $K \in D A I(X)$, segue que cada série

$$
\sum_{\alpha} a_{\alpha}\left\langle f, f_{\alpha}\right\rangle_{2} f_{\alpha}, \quad f \in L^{2}(X, \mu)
$$

converge absolutamente em $L^{2}(X, \mu)$. Portanto, cada tal série é incondicionalmente convergente em $L^{2}(X, \mu)$, ou seja, a convergência da série não depende da ordem parcial fixada ([28]). Isso completa a demonstração.

\subsection{Autovalores dos operadores gerados por séries de funções.}

Nesta seção, discutiremos alguns resultados técnicos sobre a sequência de autovalores do operador $\mathcal{K}$, ainda no caso em que $K$ tem a representação (2.0.1). 
Voltemos ao operador $\mathcal{K}_{n}$ introduzido na demonstração da Proposição 2.2.2. Como

$$
\mathcal{K}_{n}(f)(z)=\sum_{|\alpha|=0}^{n-1} a_{\alpha}\left\langle f, f_{\alpha}\right\rangle_{2} f_{\alpha}(z), \quad z \in X, \quad f \in L^{2}(X, \mu)
$$

o posto de $\mathcal{K}_{n}$ é finito e, no máximo, a cardinalidade do conjunto

$$
\left\{\alpha \in \mathbb{Z}_{+}^{m+1}:|\alpha| \leq n-1\right\}
$$

Lembrando o Lema 1.1.6 e a fórmula (1.1.6), concluímos que este posto é, no máximo, $b_{n-1}^{m+2}$. Pelo teorema da aproximação de Stirling, concluímos que

$$
b_{n-1}^{m+2}=\left(\begin{array}{c}
n+m \\
m+1
\end{array}\right) \sim \frac{n^{m+1}}{(m+1) !}, \quad(n \rightarrow \infty),
$$

e, por conseguinte,

$$
b_{n-1}^{m+2}=O\left(n^{m+1}\right), \quad(n \rightarrow \infty) .
$$

Em particular, existe o menor inteiro positivo $l$ tal que

$$
b_{n-1}^{m+2}+1 \leq(\ln )^{m+1}, \quad n=1,2, \ldots
$$

No lema a seguir, apresentamos um método para o controle de uma subsequência específica da sequência $\left\{\left|\lambda_{n}(\mathcal{K})\right|\right\}$, a qual está ordenada de acordo com o Teorema de Hilbert-Schmidt mencionado no capítulo anterior. Tal resultado é um passo técnico em direção aos resultados de decaimento para a sequência de autovalores do operador $\mathcal{K}$, por meio da análise dos coeficientes presentes na representação do núcleo.

Lema 2.3.1. Seja $K$ como em 2.0.1). Se $K$ é um elemento de $D A I(X)$, então existe um menor inteiro positivo $l$ tal que

$$
\left|\lambda_{(l n)^{m+1}}(\mathcal{K})\right| \leq \sum_{|\alpha|=n}^{\infty}\left|a_{\alpha}\right|\left\|f_{\alpha}\right\|_{2}^{2}, \quad n=1,2, \ldots
$$

Demonstração: Utilizando a representação em série garantida pela Proposição 2.2.2. podemos estimar a norma do operador $\mathcal{K}-\mathcal{K}_{n}$ como segue

$$
\begin{aligned}
\left\|\mathcal{K}-\mathcal{K}_{n}\right\| & =\sup _{\|f\|_{2}=1}\left(\int_{X}\left|\sum_{|\alpha| \geq n} a_{\alpha}\left\langle f, f_{\alpha}\right\rangle_{2} f_{\alpha}(x)\right|^{2} d \mu(x)\right)^{1 / 2} \\
& \leq \sup _{\|f\|_{2}=1}\left(\int_{X}\left(\sum_{|\alpha| \geq n}\left|a_{\alpha}\right|\left|\left\langle f, f_{\alpha}\right\rangle_{2}\right|\left|f_{\alpha}(x)\right|\right)^{2} d \mu(x)\right)^{1 / 2}, \quad n=1,2, \ldots
\end{aligned}
$$


Imitando os cálculos da demonstração da Proposição 2.2.2, obtemos

$$
\| \mathcal{K}-\mathcal{K}_{n}|| \leq\left.\sum_{|\alpha|=n}^{\infty}\left|a_{\alpha}\right||| f_{\alpha}\right|_{2} ^{2}, \quad n=1,2, \ldots
$$

Como a sequência $\left\{\left|\lambda_{n}(\mathcal{K})\right|\right\}$ é decrescente, o Teorema 1.3.10 e a fórmula 2.3 .2 combinados implicam que existe um menor inteiro positivo $l$ satisfazendo

$$
\left|\lambda_{(l n)^{m+1}}\right| \leq\left\|\mathcal{K}-\mathcal{K}_{n}\right\|, \quad n=1,2, \ldots,
$$

o que conclui a demonstração.

O teorema a seguir descreve o decaimento da sequência $\left\{\left|\lambda_{n}(\mathcal{K})\right|\right\}$, quando se tem uma informação adicional sobre o decaimento da sequência $\left\{\sum_{|\alpha|=n}^{\infty}\left|a_{\alpha}\right||| f_{\alpha} \|_{2}^{2}\right\}$.

Teorema 2.3.2. Seja $K$ como em 2.0.1). Se K é um elemento de $D A I(X)$ e

$$
\sum_{|\alpha|=n}^{\infty}\left|a_{\alpha}\right|\left\|f_{\alpha}\right\|_{2}^{2}=O\left(n^{-\gamma}\right), \quad(n \rightarrow \infty),
$$

para algum $\gamma>0$, então

$$
\left|\lambda_{n}(\mathcal{K})\right|=O\left(n^{-\gamma /(m+1)}\right), \quad(n \rightarrow \infty)
$$

Demonstração: Se $K \in D A I(X)$ e a sequência $\left\{\sum_{|\alpha|=n}^{\infty}\left|a_{\alpha}\right| \| f_{\alpha}||_{2}^{2}\right\}$ tem o decaimento descrito no enunciado do teorema, então o lema anterior nos dá que

$$
\left|\lambda_{(l n)^{m+1}}(\mathcal{K})\right| \leq \frac{M}{(\ln )^{\gamma}}, \quad n=1,2, \ldots
$$

para algum $M>0$, onde $l$ é o inteiro positivo garantido pelo lema. Uma aplicação do Lema 1.1.4 conclui a demonstração.

Observação 2.3.3. Segue do Corolário 1.3.14 que o decaimento fornecido pelo resultado acima tem relevância somente quando $2 \gamma \geq m+1$.

No resultado que fecha a seção, consideraremos o caso em que o núcleo $K$ em 2.0.1 é tal que $\left\{a_{\alpha}\right\} \subset[0, \infty)$ e $\left\{f_{\alpha}: \alpha \in \mathbb{Z}_{+}^{m+1}\right\}$ é um conjunto $L^{2}(X, \mu)$-ortogonal, ou seja,

$$
\left\langle f_{\alpha}, f_{\beta}\right\rangle_{2}=\int_{X} f_{\alpha}(z) \overline{f_{\beta}(z)} d \mu(z)=0, \quad \alpha \neq \beta
$$

Nestas condições, cada número

$$
\lambda_{\alpha}(\mathcal{K})=a_{\alpha}\left\|f_{\alpha}\right\|_{2}^{2}, \quad \alpha \in \mathbb{Z}_{+}^{m+1}
$$


é um autovalor de $\mathcal{K}$, sendo $f_{\alpha}$ a auto-função correspondente. Decorre da ortogonalidade do conjunto $\left\{f_{\alpha}: \alpha \in \mathbb{Z}_{+}^{m+1}\right\}$, que estes são exatamente os únicos autovalores não-nulos de $\mathcal{K}$. De fato, se $\mathcal{K}(h)=\lambda h$, para alguma $h \neq 0$ e $0 \neq \lambda \notin\left\{a_{\alpha}\left\langle f_{\alpha}, f_{\alpha}\right\rangle_{2}: \alpha \in \mathbb{Z}_{+}^{m+1}\right\}$, então $h$ é ortogonal a cada $f_{\alpha}$ e, consequentemente,

$$
\lambda h=\mathcal{K}(h)=\sum_{\alpha} a_{\alpha}\left\langle h, f_{\alpha}\right\rangle_{2} f_{\alpha}=0,
$$

uma contradição.

Assim sendo, estaremos particularmente interessados nas famílias $\left\{f_{\alpha}\right\}$ que são $L^{2}(X, \mu)$-ortogonais mas não são $L^{2}(X, \mu)$-ortonormais, já que no último caso, os autovalores coincidiriam com os próprios elementos da sequência $\left\{a_{\alpha}\right\}$. A relevância de uma situação como essa virá nos exemplos à frente.

Definição 2.3.4. Seja $\mathcal{H}$ um espaço de Hilbert separável. Um operador $T$ de $\mathcal{L}(\mathcal{H})$ é dito ser nuclear quando

$$
\sum_{n=1}^{\infty} s_{n}(T)<\infty .
$$

Proposição 2.3.5. Seja $K$ como em 2.0.1). Suponha que $\left\{f_{\alpha}\right\}$ é $L^{2}(X, \mu)$-ortogonal e que cada $a_{\alpha}$ é não negativo. Se $K$ é um elemento de $D A I(X)$, então $\mathcal{K}$ é nuclear.

Demonstração: Se todas as condições mencionadas no enunciado valem, o operador $\mathcal{K}$ é compacto, positivo e auto-adjunto. Logo, pelo Teorema 1.3.9, segue que

$$
s_{\alpha}(\mathcal{K})=a_{\alpha}\left\|f_{\alpha}\right\|_{2}^{2}, \quad \alpha \in \mathbb{Z}_{+}^{m+1} \text {. }
$$

Por outro lado, ainda temos que

$$
\sum_{\alpha} s_{\alpha}(\mathcal{K})=\sum_{\alpha} a_{\alpha}\left\|f_{\alpha}\right\|_{2}^{2}<\infty
$$

Isso conclui a demonstração. 



\begin{tabular}{l|c|}
\cline { 2 - 2 } & Capítulo \\
\cline { 2 - 2 } & 3 \\
\hline
\end{tabular}

\section{Decaimento dos autovalores}

Neste capítulo apresentamos resultados que descrevem o decaimento dos autovalores do operador $\mathcal{K}$, no caso em que $X$ é a esfera unitária $S^{m}$ do espaço $\mathbb{R}^{m+1}$, munida da medida de Lebesgue usual $\sigma_{m}$, o núcleo gerador $K$ em (2.0.1) pertence à classe $D A I(X)$ e

$$
f_{\alpha}(x)=x^{\alpha}, \quad x \in S^{m}, \quad \alpha \in \mathbb{Z}_{+}^{m+1} .
$$

Em seguida, utilizamos algumas fórmulas específicas para integração de polinômios homogêneos para reanalisar o problema no caso em que $X$ é a bola unitária $B^{m}=\{x \in$ $\left.\mathbb{R}^{m+1},\|x\| \leq 1\right\}$, munida da medida volume usual, mantendo-se hipóteses similares sobre os demais entes envolvidos. Ao final, alguns exemplos ilustrativos dos resultados são apresentados.

Usamos aqui a convenção usual que normalmente é utilizada em se tratando de monômios em várias variáveis: a potência nula do monômio nulo é igual a 1.

\subsection{Núcleos em séries de potências sobre $S^{m}$}

Fixada uma sequência $\left\{a_{\alpha}\right\}$ em $\mathbb{R}$, consideramos aqui o caso em que $\mathcal{K}$ é um operador integral sobre $L^{2}\left(S^{m}, \sigma_{m}\right)$, gerado por um núcleo

$$
K(x, y)=\sum_{\alpha} a_{\alpha} x^{\alpha} y^{\alpha}, \quad x, y \in S^{m}
$$


pertencente à classe $D A I\left(S^{m}\right)$. Se escrevemos $p_{\alpha}(x)=x^{\alpha}, x \in S^{m}, \alpha \in \mathbb{Z}_{+}^{m+1}$, esta última exigência torna-se

$$
\sum_{|\alpha|=0}^{\infty}\left|a_{\alpha}\right|\left\|p_{\alpha}\right\|_{2}^{2}<\infty
$$

O operador $\mathcal{K}$ é compacto, auto-adjunto e, devido ao Corolário 2.2.3, possui representação em série de potências da forma

$$
\mathcal{K}(f)=\sum_{\alpha} a_{\alpha}\left\langle f, p_{\alpha}\right\rangle_{2} p_{\alpha}, \quad f \in L^{2}\left(S^{m}, \sigma_{m}\right)
$$

com convergência na norma de $L^{2}\left(S^{m}, \sigma_{m}\right)$. Segue do teorema espectral para operadores compactos e auto-adjuntos que podemos elencar os autovalores de $\mathcal{K}$ através de uma sequência $\left\{\lambda_{n}(\mathcal{K})\right\}$ de números reais satisfazendo

$$
\left|\lambda_{1}(\mathcal{K})\right| \geq\left|\lambda_{2}(\mathcal{K})\right| \geq \cdots \geq\left|\lambda_{n}(\mathcal{K})\right| \geq \cdots
$$

Por outro lado, do Teorema 1.3.9, segue que $s_{n}(\mathcal{K})=\left|\lambda_{n}(\mathcal{K})\right|, n=1,2, \ldots$

Nos resultados que seguem, descrevemos o decaimento da sequência $\left\{\left|\lambda_{n}(\mathcal{K})\right|\right\}$ mediante algum tipo de decaimento adicional para a sequência dos coeficientes $\left\{a_{\alpha}\right\}$. Para tanto, vamos considerar a sequência $\left\{b_{k}\right\}$ de números reais positivos definida por

$$
b_{k}:=\sum_{|\alpha|=k}\left|a_{\alpha}\right|, \quad k=0,1, \ldots
$$

e ainda

$$
\|b(n)\|_{\ell_{2}}:=\left(\sum_{k=n}^{\infty} b_{k}^{2}\right)^{1 / 2}, \quad n=0,1, \ldots
$$

Observação 3.1.1. Devido ao comportamento assintótico da sequência $\left\|p_{\alpha}\right\|_{2}^{2}$ fornecido pelo Lema 1.2.6, existe uma constante $C>0$ de modo que

$$
\sum_{|\alpha|=1}^{\infty}\left|a_{\alpha}\right||| p_{\alpha}||_{2}^{2} \leq C \sum_{|\alpha|=1}^{\infty}\left|a_{\alpha}\right||\alpha|^{-m / 2}=C \sum_{k=1}^{\infty} k^{-m / 2} b_{k}
$$

Logo, a convergência da série $\sum_{k=0}^{\infty} k^{-m / 2} b_{k}$ é uma condição suficiente para que $K$ seja um elemento de $D A I\left(S^{m}\right)$.

O primeiro resultado desta seção apresenta uma relação direta entre a sequência $\left\{\|b(n)\|_{\ell^{2}}\right\}$ e uma subsequência específica de $\left\{\left|\lambda_{n}(\mathcal{K})\right|\right\}$.

Teorema 3.1.2. ( $m \geq 2)$ Se $K$ tem a representação 3.1.1) e pertence à classe $D A I\left(S^{m}\right)$, então existe um inteiro positivo l tal que

$$
\left|\lambda_{(l n)^{m+1}}(\mathcal{K})\right|=O\left(\|b(n)\|_{\ell_{2}} n^{(-m+1) / 2}\right), \quad(n \rightarrow \infty) .
$$


Demonstração: Se $K$ tem a representação 3.1.1), o Lema 2.3.1já garante a existência de um inteiro positivo $l$ tal que

$$
\left|\lambda_{(l n)^{m+1}}(\mathcal{K})\right| \leq\left.\sum_{|\alpha|=n}^{\infty}\left|a_{\alpha}\right||| p_{\alpha}\right|_{2} ^{2}, \quad n=1,2, \ldots
$$

O comportamento descrito no Teorema 1.2.6 permite a escolha de um $C>0$ tal que

$$
\sum_{|\alpha|=n}^{\infty}\left|a_{\alpha}\right|\left\|p_{\alpha}\right\|_{2}^{2} \leq C \sum_{k=n}^{\infty} k^{-m / 2} b_{k}, \quad n=1,2, \ldots
$$

Por outro lado, como a desigualdade de Hölder implica que

$$
\sum_{k=n}^{\infty} k^{-m / 2} b_{k} \leq\left(\sum_{k=n}^{\infty} k^{-m}\right)^{1 / 2}\left(\sum_{k=n}^{\infty} b_{k}^{2}\right)^{1 / 2}, \quad n=1,2, \ldots
$$

o resultado segue como consequência do Lema 1.1 .3 e da restrição $m \geq 2$.

Corolário 3.1.3. Nas condições do teorema anterior, se $b_{k}=O\left(k^{-\gamma}\right),(k \rightarrow \infty)$, para algum $\gamma>1 / 2$, então

$$
\left|\lambda_{n}(\mathcal{K})\right|=O\left(n^{-(2 \gamma-2+m) / 2(m+1)}\right), \quad(n \rightarrow \infty)
$$

Demonstração: Seja $\gamma>1 / 2$ tal que $b_{k}=O\left(k^{-\gamma}\right),(k \rightarrow \infty)$. Note que isso já implica que $K \in D A I\left(S^{m}\right)$. Pelo Lema 1.1.3, podemos escrever

$$
\|b(n)\|_{\ell_{2}}=\left(\sum_{k=n}^{\infty} b_{k}^{2}\right)^{1 / 2} \leq M\left(\sum_{k=n}^{\infty} k^{-2 \gamma}\right)^{1 / 2} \leq M_{1} n^{-\gamma+1 / 2}, \quad n=1,2, \ldots,
$$

para constantes $M$ e $M_{1}$ convenientes. Retornando ao Teorema 3.1 .2 obtemos

$$
\left|\lambda_{(l n)^{m+1}}(\mathcal{K})\right|=O\left(n^{-\gamma+1-m / 2}\right), \quad(n \rightarrow \infty),
$$

para algum inteiro positivo $l$. Segue do Lema 1.1.4 que podemos escolher $M_{2}>0$ satisfazendo

$$
\left|\lambda_{n}(\mathcal{K})\right| \leq M_{2} n^{-(2 \gamma-2+m) / 2(m+1)}, \quad n=1,2, \ldots
$$

Isto conclui a demonstração.

Observação 3.1.4. O Corolário 1.3 .14 já garante o decaimento básico $\left|\lambda_{n}(\mathcal{K})\right|=$ $o\left(n^{-1 / 2}\right)$, quando $n \rightarrow \infty$. Logo, o resultado acima só é relevante no caso $\gamma>3 / 2$. Particularmente, note que quando $\gamma>2+m / 2$, o decaimento acima se mostra mais rápido do que o garantido nas condições do Teorema de Mercer $([18,[50])$. 
Teorema 3.1.5. Seja $K$ como em 3.1.1). Suponha que exista $c \in(0,1)$ e um inteiro positivo $N$ de modo que

$$
\frac{b_{k+1}}{b_{k}} \leq c, \quad k=N, N+1, \ldots
$$

Então existe um inteiro positivo l tal que

$$
\left|\lambda_{(l n)^{m+1}}(\mathcal{K})\right|=O\left(b_{n} n^{-m / 2}\right), \quad(n \rightarrow \infty) .
$$

Demonstração: Primeiramente, note que a hipótese sobre a sequência $\left\{b_{k}\right\}$ garante que $K \in D A I\left(S^{m}\right)$ (veja Observação 3.1.1). Já o Lema 2.3.1, garante a existência de um inteiro positivo $l$ tal que

$$
\left|\lambda_{(l n)^{m+1}}(\mathcal{K})\right| \leq \sum_{|\alpha|=n}^{\infty}\left|a_{\alpha}\right||| p_{\alpha} \|_{2}^{2}, \quad n=1,2, \ldots,
$$

enquanto que dos Lemas 1.2 .5 e 1.1 .7 obtemos

$$
\begin{aligned}
\sum_{|\alpha|=n}^{\infty}\left|a_{\alpha}\right|\left\|p_{\alpha}\right\|_{2}^{2} & =2 \pi^{(m+1) / 2} \sum_{|\alpha|=n}^{\infty} \frac{\left|a_{\alpha}\right|(2 \alpha) !}{4^{|\alpha|} \alpha ! \Gamma(|\alpha|+(m+1) / 2)} \\
& \leq 2 \pi^{(m+1) / 2} \sum_{k=0}^{\infty} \frac{(2 n+2 k) ! b_{n+k}}{4^{n+k}(n+k) ! \Gamma(n+k+(m+1) / 2)}, \quad n=1,2 \ldots
\end{aligned}
$$

Por outro lado, a hipótese sobre os $b_{k}$ implica que

$$
b_{n+k} \leq c^{k} b_{n}, \quad k=0,1, \ldots, \quad n=N, N+1, \ldots,
$$

enquanto que o teorema da aproximação de Stirling nos garante que, fixado $n \geq 1$,

$$
\frac{(2 n+2 k) !}{4^{n+k}(n+k) ! \Gamma(n+k+(m+1) / 2)} \sim \frac{\pi^{-1 / 2}}{(n+k)^{m / 2}}, \quad(k \rightarrow \infty) .
$$

Combinando as informações acima, fica claro que podemos escolher uma constante $C>0$ satisfazendo

$$
\left|\lambda_{(l n)^{m+1}}(\mathcal{K})\right| \leq C b_{n} \sum_{k=0}^{\infty} \frac{c^{k}}{(n+k)^{m / 2}} \leq C\left(\sum_{k=0}^{\infty} c^{k}\right) \frac{b_{n}}{n^{m / 2}}, \quad n=N+1, N+2, \ldots
$$

A demonstração está finalizada.

Especificando um pouco mais o decaimento da sequência $\left\{b_{k}\right\}$, chegamos a um decaimento para toda a sequência de autovalores de $\mathcal{K}$.

Corolário 3.1.6. Nas condições do teorema anterior, suponha adicionalmente que $b_{n}=O\left(n^{-\gamma}\right),(n \rightarrow \infty)$, para algum $\gamma>0$. Então

$$
\left|\lambda_{n}(\mathcal{K})\right|=O\left(n^{-(2 \gamma+m) / 2(m+1)}\right), \quad(n \rightarrow \infty) .
$$


Demonstração: Com a hipótese adicional sobre $\left\{b_{n}\right\}$, a desigualdade no enunciado do teorema anterior toma a forma

$$
\left|\lambda_{(l n)^{m+1}}(\mathcal{K})\right| \leq \frac{C_{1}}{(\ln )^{\gamma+m / 2}}, \quad n=1,2, \ldots,
$$

para alguma constante positiva $C_{1}$ dependendo somente de $l, m$ e $c$. Uma aplicação do Lema 1.1.4 nos leva a

$$
\left|\lambda_{n}(\mathcal{K})\right| \leq \frac{C_{2}}{n^{(2 \gamma+m) / 2(m+1)}}, \quad n=1,2, \ldots,
$$

para alguma constante positiva $C_{2}$.

Novamente, observamos que o resultado acima é relevante somente no caso em que $\gamma \geq(m+2) / 2$.

O próximo resultado é uma variante ligeiramente mais fraca do teorema anterior. Ela é baseada em uma hipótese mais modesta sobre a sequência $\left\{b_{k}\right\}$ e exige uma restrição maior na dimensão $m$.

Teorema 3.1.7. $(m \geq 3)$ Seja $K$ como em 3.1.1). Se $\left\{b_{k}\right\}$ é não-crescente, então existe um inteiro positivo l tal que

$$
\left|\lambda_{(l n)^{m+1}}(\mathcal{K})\right|=O\left(b_{n} n^{-(m-2) / 2}\right), \quad(n \rightarrow \infty) .
$$

Demonstração: Se $\left\{b_{k}\right\}$ é não-crescente e $m \geq 3$, então $K \in D A I\left(S^{m}\right)$. Agora, podemos modificar as estimativas feitas na demonstração do Teorema 3.1.5 e obter uma constante $M>0$ de modo que

$$
\begin{aligned}
\sum_{|\alpha|=n}^{\infty}\left|a_{\alpha}\right||| p_{\alpha} \|_{2}^{2} & \leq 2 \pi^{(m+1) / 2} \sum_{k=n}^{\infty} \frac{(2 k) ! b_{k}}{4^{k} k ! \Gamma(k+(m+1) / 2)} \\
& \leq 2 M \pi^{m / 2} b_{n} \sum_{k=n}^{\infty} \frac{1}{k^{m / 2}}, \quad n=1,2, \ldots
\end{aligned}
$$

Particularmente, como $m \geq 3$, podemos aplicar o Lema 1.1 .3 obtendo

$$
\sum_{|\alpha|=n}^{\infty}\left|a_{\alpha}\right|\left\|p_{\alpha}\right\|_{2}^{2} \leq M_{1} b_{n} n^{-(m-2) / 2}, \quad n=1,2, \ldots
$$

para alguma constante $M_{1}>0$, o que conclui a demonstração.

Corolário 3.1.8. Nas condições do teorema anterior, se $b_{n}=O\left(n^{-\gamma}\right),(n \rightarrow \infty)$, para algum $\gamma>0$, então

$$
\left|\lambda_{n}(\mathcal{K})\right|=O\left(n^{-(2 \gamma+m-2) / 2(m+1)}\right), \quad(n \rightarrow \infty)
$$


Demonstração: Combinando a hipótese com o teorema anterior, podemos escolher $M>0$ tal que

$$
\left|\lambda_{(l n)^{m+1}}(\mathcal{K})\right| \leq M n^{-(2 \gamma+m-2) / 2}, \quad n=1,2, \ldots .
$$

O resultado é obtido com uma aplicação do Lema 1.1.4.

Note que a hipótese sobre a sequência $\left\{b_{k}\right\}$ no Teorema 3.1 .5 implica que

$$
\limsup _{k \rightarrow \infty} \frac{b_{k+1}}{b_{k}}<1
$$

Disto podemos concluir que ([33, p. 58]),

$$
\sum_{|\alpha|=0}^{\infty}\left|a_{\alpha}\right|=\sum_{n=0}^{\infty} b_{n}<\infty
$$

Particularmente, $\sum_{\alpha} a_{\alpha}<\infty$, e $K$ é então contínua em $S^{m} \times S^{m}$.

\subsection{Núcleos em séries de potências sobre $B^{m}$}

Nesta seção, buscamos estender a análise feita na seção anterior, substituindo $S^{m}$ pela bola unitária $B^{m}=\left\{x \in \mathbb{R}^{m+1}:\|x\| \leq 1\right\}$ e $d \sigma_{m}(x)$ pelo elemento de volume $d x$.

O ponto inicial e talvez motivador para os resultados aqui descritos é a seguinte fórmula que relaciona integração sobre $S^{m}$ com integração sobre $B^{m}([8])$.

Lema 3.2.1. Se f é uma função contínua e homogênea de grau $\rho$ em $\mathbb{R}^{m+1}$, então

$$
\int_{B^{m}} f(y) d y=\frac{1}{\rho+m+1} \int_{S^{m}} f(x) d \sigma_{m}(x) .
$$

Lembramos que homogeneidade de grau $\rho$, refere-se à propriedade

$$
f(r y)=r^{\rho} f(y), \quad y \in \mathbb{R}^{m+1}, \quad r \geq 0
$$

Aplicando esta fórmula no caso em que $f$ é um monômio e lembrando da Proposição 1.2.5 temos:

Proposição 3.2.2. Se $\alpha$ é um multi-índice de $\mathbb{Z}_{+}^{m+1}$, então

$$
\int_{B^{m}} y^{2 \alpha} d y=\frac{2}{2|\alpha|+m+1} \frac{(2 \alpha) ! \pi^{(m+1) / 2}}{4^{|\alpha|} \alpha ! \Gamma(|\alpha|+(m+1) / 2)}
$$

Definindo,

$$
\hat{p}_{\alpha}(y)=y^{\alpha}, \quad y \in B^{m}, \quad \alpha \in \mathbb{Z}_{+}^{m+1},
$$


também concluímos que

$$
\left\|\hat{p}_{\alpha}\right\|_{2}^{2}=\int_{B^{m}}\left|y^{\alpha}\right|^{2} d y=\frac{1}{2|\alpha|+m+1} \int_{S^{m}}\left|x^{\alpha}\right|^{2} d \sigma_{m}(x)=\frac{\left\|p_{\alpha}\right\|_{2}^{2}}{2|\alpha|+m+1}, \quad \alpha \in \mathbb{Z}_{+}^{m+1} .
$$

Logo, a fórmula

$$
\left\|\hat{p}_{\alpha}\right\|_{2}^{2}=O\left(\frac{1}{|\alpha|^{1+m / 2}}\right), \quad(|\alpha| \rightarrow \infty)
$$

é uma versão daquela descrita no Teorema 1.2.6 para o contexto que estamos discutindo agora.

Em vista do exposto acima, voltamos nossa atenção para um núcleo gerador da forma

$$
\hat{K}(x, y)=\sum_{\alpha} a_{\alpha} x^{\alpha} y^{\alpha}, \quad x, y \in B^{m}
$$

com $\left\{a_{\alpha}\right\} \subset \mathbb{R}$ e $\hat{K} \in D A I\left(B^{m}\right)$. A representação em série, análoga aquela garantida pelo Corolário 2.2.3, para o operador

$$
\hat{\mathcal{K}}(f)(x)=\int_{B^{m}}\left(\sum_{\alpha} a_{\alpha} x^{\alpha} y^{\alpha}\right) f(y) d y, \quad x \in B^{m}, \quad f \in L^{2}\left(B^{m}\right),
$$

toma a forma

$$
\hat{\mathcal{K}}(f)=\sum_{\alpha} a_{\alpha}\left\langle f, \hat{p}_{\alpha}\right\rangle_{2} p_{\alpha}, \quad f \in L^{2}\left(B^{m}\right) .
$$

Mantendo a mesma notação para a sequência $\left\{b_{k}\right\}$, note que uma condição suficiente para se ter $\hat{K} \in D A I\left(B^{m}\right)$ é que a sequência $\left\{b_{k} k^{-1-m / 2}\right\}$ seja somável. De fato, segue de (3.2.1), que podemos escolher uma constante $C>0$ de modo que

$$
\left.\sum_{\alpha}\left|a_{\alpha}\right||| \hat{p}_{\alpha}\right|_{2} ^{2} \leq C \sum_{\alpha}\left|a_{\alpha}\right||\alpha|^{-1-m / 2}=C \sum_{k=0}^{\infty} k^{-1-m / 2} b_{k}
$$

Nas condições acima, a Proposição 2.2.1 garante que $\hat{\mathcal{K}}$ é compacto, autoadjunto e os valores singulares de $\hat{\mathcal{K}}$ podem ser dispostos em uma sequência decrescente da mesma maneira que fizemos para os valores singulares de $\mathcal{K}$.

A seguir, seguem as versões dos resultados da seção anterior adaptados ao contexto atual. Note que as restrições sobre a dimensão $m$ exigidas no caso esférico já não são mais necessárias, uma vez que a norma $\left\|\hat{p}_{\alpha}\right\|_{2}$ decai mais rapidamente do que $\left\|p_{\alpha}\right\|_{2}$, quando $|\alpha| \rightarrow \infty$.

Teorema 3.2.3. Seja $\hat{K}$ como em 3.2.2) e pertencente à classe $D A I\left(B^{m}\right)$. Então existe um inteiro positivo l tal que

$$
\left|\lambda_{(l n)^{m+1}}(\hat{\mathcal{K}})\right|=O\left(\|b(n)\|_{\ell_{2}} n^{-(m+1) / 2}\right), \quad(n \rightarrow \infty) .
$$


Demonstração: Segue de uma versão espelhada no Teorema 3.1 .2 para o caso atual, utilizando a estimativa assintótica de $\left\|\hat{p}_{\alpha}\right\|_{2}^{2}$ descrita em 3.2.1) .

Corolário 3.2.4. Nas condições do teorema anterior, se existe $\gamma>1 / 2$ satisfazendo $b_{k}=O\left(k^{-\gamma}\right),(k \rightarrow \infty)$, então

$$
\left|\lambda_{n}(\hat{\mathcal{K}})\right|=O\left(n^{-(2 \gamma+m) / 2(m+1)}\right), \quad(n \rightarrow \infty) .
$$

Demonstração: Seja $\gamma>1 / 2$ tal que $b_{k}=O\left(k^{-\gamma}\right),(k \rightarrow \infty)$. O Lema 1.1 .3 permite encontrarmos constantes positivas $M$ e $M_{1}$ de modo que

$$
\|b(n)\|_{\ell_{2}}=\left(\sum_{k=n}^{\infty} b_{k}^{2}\right)^{1 / 2} \leq M\left(\sum_{k=n}^{\infty} k^{-2 \gamma}\right)^{1 / 2} \leq M_{1} n^{-\gamma+1 / 2}, \quad n=1,2, \ldots
$$

pois $\gamma>1 / 2$. Combinando esta desigualdade com a estimativa dada no teorema anterior obtemos

$$
\left|\lambda_{(l n)^{m+1}}(\hat{\mathcal{K}})\right|=O\left(n^{-\gamma-m / 2}\right), \quad(n \rightarrow \infty) .
$$

Do Lema 1.1.4, podemos escolher $M_{2}>0$ satisfazendo

$$
\left|\lambda_{n}(\hat{\mathcal{K}})\right| \leq M_{2} n^{-(2 \gamma+m) / 2(m+1)}, \quad n=1,2, \ldots
$$

Isto conclui a demonstração.

Teorema 3.2.5. Seja $\hat{K}$ como em (3.2.2). Suponha que exista $c \in(0,1)$ e um inteiro positivo $N$ de modo que

$$
\frac{b_{k+1}}{b_{k}} \leq c, \quad k=N, N+1, \ldots
$$

Então existe um inteiro positivo l tal que

$$
\left|\lambda_{(l n)^{m+1}}(\hat{\mathcal{K}})\right|=O\left(b_{n} n^{-1-m / 2}\right), \quad(n \rightarrow \infty) .
$$

Demonstração: A demonstração é uma cópia daquela do Teorema 3.1.5, utilizando a estimativa para $\left\|\hat{p}_{\alpha}\right\|_{2}^{2}$ dada em 3.2.1.

Corolário 3.2.6. Nas condições do teorema anterior, suponha adicionalmente que exista $\gamma \in \mathbb{R}$ tal que $b_{n}=O\left(n^{-\gamma}\right),(n \rightarrow \infty)$. Então,

$$
\left|\lambda_{n}(\hat{\mathcal{K}})\right|=O\left(n^{-(2+2 \gamma+m) / 2(m+1)}\right), \quad(n \rightarrow \infty) .
$$


Demonstração: Análoga à demonstração do Corolário 3.1.6.

Novamente, o decaimento básico garantido pelo Corolário 1.3.14 nos mostra que a informação acima é relevante somente quando $\gamma \geq-1 / 2$. No entanto, nas condições do Teorema, já temos garantido $\gamma \geq 1$.

Teorema 3.2.7. Seja $\hat{K}$ como em (3.2.2). Se $\left\{b_{k}\right\}$ é não-crescente, então existe um inteiro positivo l tal que

$$
\left|\lambda_{(l n)^{m+1}}(\hat{\mathcal{K}})\right|=O\left(b_{n} n^{-m / 2}\right), \quad(n \rightarrow \infty) .
$$

Demonstração: Se $\left\{b_{k}\right\}$ é não-crescente, segue que $\hat{K} \in D A I\left(B^{m}\right)$. Procedendo de forma análoga à demonstração do Teorema 3.1.7 obtemos

$$
\sum_{|\alpha|=n}^{\infty}\left|a_{\alpha}\right|\left\|\hat{p}_{\alpha}\right\|_{2}^{2} \leq M b_{n} \sum_{k=n}^{\infty} \frac{1}{k^{1+m / 2}}, \quad n=1,2, \ldots
$$

para alguma constante $M>0$. O resultado segue do Lema 1.1.3.

Note que o resultado acima, diferentemente de sua versão na esfera (Teorema 3.1.7), é válido para todo $m \geq 1$. No entanto, a estimativa final também é mais fraca do que a obtida no Teorema 3.2 .3 .

O corolário a seguir se baseia em um decaimento específico para a sequência $\left\{b_{n}\right\}$.

Corolário 3.2.8. Nas condições acima, se $b_{n}=O\left(n^{-\gamma}\right),(n \rightarrow \infty)$, para algum $\gamma>0$, então

$$
\left|\lambda_{n}(\hat{\mathcal{K}})\right|=O\left(n^{-(m+2 \gamma) / 2(m+1)}\right), \quad(n \rightarrow \infty) .
$$

Demonstração: O teorema anterior fornece o decaimento

$$
\left|\lambda_{(l n)^{m+1}}(\hat{\mathcal{K}})\right|=O\left(b_{n} n^{-m / 2}\right), \quad(n \rightarrow \infty),
$$

para algum inteiro positivo $l$. Combinando isto com a hipótese e o Lema 1.1.4 deduzimos o decaimento fornecido pelo corolário.

\subsection{Alguns exemplos}

Esta seção tem por objetivo ilustrar alguns dos resultados anteriores. Como veremos, apesar de alguns dos resultados que obtivemos descreverem tão somente um certo controle para uma subsequência específica da sequência de valores singulares do operador integral, o controle da sequência toda só demanda um pouco mais de esforço. Esta faceta também é ratificada nos exemplos. 
Exemplo 3.3.1. Utilizando a definição da sequência $\left\{b_{k}\right\}$ e o comportamento assintótico de $\left\{\left\|p_{\alpha}\right\|_{2}\right\}$, vimos que existe uma constante $C>0$ satisfazendo

$$
\sum_{|\alpha|=n}^{\infty}\left|a_{\alpha}\right||| p_{\alpha} \|_{2}^{2} \leq C \sum_{k=n}^{\infty} b_{k} k^{-m / 2}, \quad n=1,2, \ldots
$$

Suponha que exista $\delta \in \mathbb{R}$ tal que

$$
b_{k} \sim k^{\delta}, \quad(k \rightarrow \infty) .
$$

Então, para $n$ suficientemente grande, vale a desigualdade

$$
\sum_{|\alpha|=n}^{\infty}\left|a_{\alpha}\right|\left\|p_{\alpha}\right\|_{2}^{2} \leq C_{1} \sum_{k=n}^{\infty} k^{\delta-m / 2}, \quad n=1,2, \ldots,
$$

contanto que $C_{1}>0$ seja suficientemente grande. Se $\delta-m / 2<-1$, ou seja, se $\delta \in(-\infty,(m-2) / 2)$, então obtemos do Lema 1.1.3 que

$$
\sum_{|\alpha|=n}^{\infty}\left|a_{\alpha}\right|\left\|p_{\alpha}\right\|_{2}^{2}=O\left(n^{\delta+1-m / 2}\right), \quad(n \rightarrow \infty) .
$$

Assim, uma aplicação do Teorema 2.3.2 nos garante a seguinte estimativa

$$
\left|\lambda_{n}(\mathcal{K})\right|=O\left(n^{(2 \delta+2-m) / 2(m+1)}\right), \quad(n \rightarrow \infty) .
$$

No entanto, a Observação 2.3.3 assegura a relevância deste último decaimento somente quando $(2 \delta+2-m) / 2(m+1) \leq-1 / 2$, ou seja, quando $\delta \leq-3 / 2$.

No caso da bola $B^{m}$, começamos com a desigualdade

$$
\sum_{|\alpha|=n}^{\infty}\left|a_{\alpha}\right| \mid \hat{p}_{\alpha} \|_{2}^{2} \leq C \sum_{k=n}^{\infty} b_{k} k^{-1-m / 2}, \quad n=1,2, \ldots
$$

Supondo a mesma hipótese para a sequência $\left\{b_{k}\right\}$, podemos escrever

$$
\sum_{|\alpha|=n}^{\infty}\left|a_{\alpha}\right| \|\left.\hat{p}_{\alpha}\right|_{2} ^{2} \leq C \sum_{k=n}^{\infty} k^{\delta-1-m / 2}, \quad n=1,2, \ldots,
$$

para algum $C>0$. Se $\delta-1-m / 2<-1$, ou seja, $\delta<m / 2$, o Lema 1.1 .3 é aplicável e

$$
\sum_{|\alpha|=n}^{\infty}\left|a_{\alpha}\right|\left\|\hat{p}_{\alpha}\right\|_{2}^{2}=O\left(n^{\delta-m / 2}\right), \quad(n \rightarrow \infty) .
$$

O Teorema 2.3.2 garante então o seguinte decaimento

$$
\left|\lambda_{n}(\hat{\mathcal{K}})\right|=O\left(n^{(2 \delta-m) / 2(m+1)}\right), \quad(n \rightarrow \infty) .
$$

Este último é significativo no caso em que $\delta \leq-1 / 2$. 
Os exemplos e estimativas que seguem serão consideradas no caso da esfera $S^{m}$ apenas. Entretanto, como indicado na seção anterior, todos eles podem ser adaptados para o caso em que a esfera é substituída por $B^{m}$.

Exemplo 3.3.2. Sejam $p>0$ e

$$
a_{\alpha}=\frac{|\alpha| !}{|\alpha|^{p}(m+1)^{|\alpha|} \alpha !}, \quad \alpha \in \mathbb{Z}_{+}{ }^{m+1} .
$$

Utilizando o Teorema 1.1.9, a expressão definindo $K$ toma a forma

$$
K(x, y)=\sum_{n=0}^{\infty} \frac{1}{n^{p}(m+1)^{n}} \sum_{|\alpha|=n} \frac{|\alpha| !}{\alpha !} x^{\alpha} y^{\alpha}=\sum_{n=0}^{\infty} \frac{(x \cdot y)^{n}}{n^{p}(m+1)^{n}}, \quad x, y \in S^{m} .
$$

Segue do Corolário 1.1 .10 que a sequência $\left\{b_{n}\right\}$ é determinada pela fórmula

$$
b_{n}=\sum_{|\alpha|=n} \frac{|\alpha| !}{|\alpha|^{p}(m+1)^{|\alpha|} \alpha !}=\frac{1}{n^{p}(m+1)^{n}} \sum_{|\alpha|=n} \frac{|\alpha| !}{\alpha !}=\frac{1}{n^{p}}, \quad n=1,2, \ldots
$$

É evidente então que $\left\{b_{k}\right\}$ é decrescente e que

$$
b_{n}=O\left(n^{-p}\right), \quad(n \rightarrow \infty) .
$$

Quando $m \geq 3$, podemos aplicar o Teorema 3.1.7 e, em seguida, o Corolário 3.1.8, validando o decaimento

$$
\lambda_{n}(\mathcal{K})=O\left(n^{-(2 p+m-2) / 2(m+1)}\right), \quad(n \rightarrow \infty)
$$

Note que quando $p>(m+4) / 2$, obtemos

$$
\lambda_{n}(\mathcal{K})=O\left(n^{-s}\right), \quad(n \rightarrow \infty),
$$

para algum $s=s(p, m)>1$.

Exemplo 3.3.3. Analisemos agora o caso em que

$$
a_{\alpha}=\frac{1}{\alpha !}, \quad \alpha \in \mathbb{Z}_{+}^{m+1} .
$$

Neste caso, o núcleo $K$ é dado por

$$
K(x, y)=\sum_{\alpha} \frac{1}{\alpha !} x^{\alpha} y^{\alpha}=\exp (x \cdot y), \quad x, y \in S^{m} .
$$

Usando o Corolário 1.1.10 para explicitar os termos da sequência $\left\{b_{n}\right\}$, deduzimos que

$$
b_{n}=\sum_{|\alpha|=n} \frac{1}{\alpha !}=\frac{1}{n !} \sum_{|\alpha|=n} \frac{n !}{\alpha !}, \quad n=0,1, \ldots
$$


ou seja,

$$
b_{n}=\frac{(m+1)^{n}}{n !} \quad n=0,1, \ldots
$$

Consequentemente,

$$
\frac{b_{n+1}}{b_{n}}<\frac{m+1}{m+2}<1, \quad n=m+1, m+2, \ldots
$$

Por outro lado, utilizando o teorema da aproximação de Stirling obtemos,

$$
b_{n}=\frac{(m+1)^{n}}{n !} \sim \frac{(m+1)^{n} e^{n}}{\sqrt{2 \pi} n^{n+1 / 2}}, \quad(n \rightarrow \infty)
$$

Segue do Teorema 3.1 .5 que existe uma constante $C>0$ tal que

$$
\lambda_{(l n)^{m+1}}(\mathcal{K}) \leq \frac{C p^{n}}{n^{n+(m+1) / 2}}, \quad n=N, N+1, \ldots,
$$

para $N$ suficientemente grande e $p=e(m+1)$. Reescrevendo,

$$
\lambda_{(l n)^{m+1}}(\mathcal{K}) \leq \frac{C l^{n+(m+1) / 2} p^{n}}{(\ln )^{n+(m+1) / 2}}=\frac{C_{1} p_{1}^{n}}{(\ln )^{n+(m+1) / 2}}, \quad n=N, N+1, N+2, \ldots,
$$

onde $p_{1}=l p$ e $C_{1}=C l^{(m+1) / 2}$. Agora, observe que a monotonicidade da sequência $\left\{\lambda_{n}(\mathcal{K})\right\}$ implica que, para cada $j \in\{0,1, \ldots, l-1\}$,

$$
\lambda_{(\ln +j)^{m+1}}(\mathcal{K}) \leq C_{1} \frac{p_{1}^{n}}{(\ln +j)^{n+(m+1) / 2}} \frac{(\ln +j)^{n+(m+1) / 2}}{(\ln )^{n+(m+1) / 2}}, \quad n=N+1, N+2, \ldots,
$$

Como para cada tal $j$,

$$
\frac{(\ln +j)^{n+(m+1) / 2}}{(\ln )^{n+(m+1) / 2}}=\left(1+\frac{j}{\ln }\right)^{n+(m+1) / 2} \leq\left(1+\frac{l-1}{\ln }\right)^{n+(m+1) / 2}
$$

e

$$
\left(1+\frac{l-1}{l n}\right)^{n+(m+1) / 2} \sim e^{1-1 / l}, \quad(n \rightarrow \infty),
$$

segue que o termo da esquerda na desigualdade acima é limitado para todo $j \in$ $\{0,1, \ldots, l-1\}$ e todo $n \geq N$. Portanto, existe constante $C_{2}>0$ tal que

$$
\lambda_{(l n+j)^{m+1}}(\mathcal{K}) \leq C_{2} \frac{p_{1}^{n}}{(\ln +j)^{n+(m+1) / 2}}, \quad n=N, N+1, \ldots, \quad j=0,1, \ldots, l-1,
$$

ou seja,

$$
\lambda_{n^{m+1}}(\mathcal{K}) \leq C_{2} \frac{p_{1}^{n}}{n^{n+(m+1) / 2}}, \quad n=N, N+1, \ldots
$$

Para buscar um controle para toda a sequência $\left\{\lambda_{n}(\mathcal{K})\right\}$, fixemos arbitrariamente $n>$ $N$. Por argumentos de monotonicidade, segue que para cada $j=0,1, \ldots,(n+1)^{m+1}-$ $n^{m+1}-1$

$$
\lambda_{n^{m+1}+j}(\mathcal{K}) \leq C_{2} \frac{p_{1}^{n}}{\left(n^{m+1}+j\right)^{\frac{n}{m+1}+\frac{1}{2}}}\left(\frac{n^{m+1}+j}{n^{m+1}}\right)^{\frac{n}{m+1}+\frac{1}{2}}
$$


Por outro lado, como

$(n+1)^{m+1}-n^{m+1}-1 \leq n^{m+1}\left(1+\frac{1}{n}\right)^{m+1}-n^{m+1} \leq n^{m+1}\left(2^{m+1}-1\right), \quad n=1,2, \ldots$,

segue que

$$
\begin{aligned}
\left(\frac{n^{m+1}+j}{n^{m+1}}\right)^{\frac{n}{m+1}+\frac{1}{2}} & =\left(1+\frac{j}{n^{m+1}}\right)^{\frac{n}{m+1}+\frac{1}{2}} \\
& \leq\left(1+2^{m+1}-1\right)^{\frac{n}{m+1}+\frac{1}{2}}=2^{n+(m+1) / 2}, \quad n=1,2, \ldots
\end{aligned}
$$

Logo, existe $C_{3}>0$ de modo que

$$
\lambda_{n^{m+1}+j}(\mathcal{K}) \leq C_{3} \frac{p_{2}^{n}}{\left(n^{m+1}+j\right)^{\frac{n}{m+1}+\frac{1}{2}}}, j=0,1, \ldots,(n+1)^{m+1}-n^{m+1}-1,
$$

onde $p_{2}=2 p_{1}$. Portanto,

$$
\lambda_{n}(\mathcal{K}) \leq C_{3} \frac{p_{2}^{n}}{n^{\frac{n}{m+1}+\frac{1}{2}}}, \quad n=N, N+1, \ldots,
$$

onde $p_{2}=l e(m+1)$. Em particular, $\lambda_{n}(\mathcal{K})=O\left(n^{-\gamma}\right),(n \rightarrow \infty)$, para todo $\gamma>$ 0 . Conforme [47, p. 139], $\left\{\lambda_{n}(\mathcal{K})\right\}$ é então uma sequência rapidamente decrescente. Particularmente, em [7] são apresentadas estimativas finas para estes autovalores.

Exemplo 3.3.4. Seja $r$ um número real e definamos

$$
a_{\alpha}=r^{-|\alpha|}|\alpha|^{-m / 2}, \quad \alpha \in \mathbb{Z}_{+}^{m+1} .
$$

Pelo Lema 1.1.6, temos que

$$
b_{n}=\sum_{|\alpha|=n}\left|a_{\alpha}\right|=\sum_{|\alpha|=n}|r|^{-|\alpha|}|\alpha|^{-m / 2}=|r|^{-n} n^{-m / 2}\left(\begin{array}{c}
m+n \\
m
\end{array}\right), \quad n=0,1, \ldots
$$

Pelo teorema da aproximação de Stirling, obtemos

$$
b_{n} \sim \frac{1}{m !}|r|^{-n} n^{m / 2}, \quad(n \rightarrow \infty) .
$$

Consequentemente,

$$
\frac{b_{n+1}}{b_{n}} \sim \frac{1}{|r|}\left(\frac{n}{n+1}\right)^{m / 2} \rightarrow \frac{1}{|r|}, \quad(n \rightarrow \infty) .
$$

Se $|r|>1$, podemos escolher $N$ e $c \in(0,1)$ de modo que

$$
\frac{b_{n+1}}{b_{n}} \leq c, \quad n=N, N+1, \ldots
$$


Daí, o Teorema 3.1.5 garante a existência de $M>0$ tal que

$$
\left|\lambda_{(l n)^{m+1}}(\mathcal{K})\right| \leq M|r|^{-n}, \quad n=1,2, \ldots
$$

Como

$$
\begin{aligned}
\left|\lambda_{(l n+j)^{m+1}}(\mathcal{K})\right|^{l} & \leq M^{l}|r|^{l-1}|r|^{-(l n+j)} \\
& =M^{l}|r|^{-(l n+j)}, \quad j=0,1, \ldots, l-1, \quad n=N, N+1, \ldots
\end{aligned}
$$

segue que $\left|\lambda_{n^{m+1}}(\mathcal{K})\right| \leq M^{l}|r|^{-n / l}, n=N, N+1, \ldots$ Ainda

$$
\begin{aligned}
\left|\lambda_{n^{m+1}+j}(\mathcal{K})\right| & \leq M^{l}|r|^{-n / l} \\
& =M^{l}|r|^{-\frac{1}{2 l}\left(n^{m+1}+j\right)^{\frac{1}{m+1}}}|r|^{-n / l}|r|^{\frac{1}{2 l}\left(n^{m+1}+j\right)^{\frac{1}{m+1}}}
\end{aligned}
$$

para $j \in\left\{0,1, \ldots,(n+1)^{m+1}-n^{m+1}-1\right\}$ e $n=N, N+1, \ldots$. Por outro lado, a desigualdade

$$
n^{m+1}+j \leq(n+1)^{m+1}-1 \leq(2 n)^{m+1}
$$

implica que

$$
\left|\lambda_{n^{m+1}+j}(\mathcal{K})\right| \leq M^{l}|r|^{-\frac{1}{2 l}\left(n^{m+1}+j\right)^{\frac{1}{m+1}}}|r|^{-n / l}|r|^{\frac{1}{2 l} 2 n}=M^{l}|r|^{-\frac{1}{2 l}\left(n^{m+1}+j\right)^{\frac{1}{m+1}}}
$$

para os mesmos valores de $j$ e $n$. Em outras palavras,

$$
\left|\lambda_{n}(\mathcal{K})\right|=O\left(|r|^{-\frac{n^{\frac{1}{m+1}}}{2 l}}\right), \quad(n \rightarrow \infty) .
$$


Capítulo

4

\section{Núcleos definidos por expansões ortogonais.}

Aqui, continuamos com o estudo iniciado no capítulo anterior, agora investigando o decaimento de autovalores do operador $\mathcal{K}$, no caso em que $X$ é um subconjunto não-vazio de $\mathbb{R}^{m+1}$ ou $\mathbb{C}^{m+1}$, munido da medida de Lebesgue $\nu$, e $\left\{f_{\alpha}\right\}$ em 2.0.1 é um conjunto $L^{2}(X, \nu)$-ortogonal. Os decaimentos apresentados são alcançados ao custo de hipóteses sobre as sequências $\left\{a_{\alpha}\right\}$ e $\left\{\left\|f_{\alpha}\right\|_{2}\right\}$. Os casos particulares em que $K$ tem representação em série de potências 2 (2.0.2) sobre ambos, a esfera complexa $\Omega_{m}$ e a bola complexa $\Delta_{m}$ de $\mathbb{C}^{m+1}$, são discutidos separadamente.

\subsection{Resultados gerais}

Nesta seção, pretendemos analisar o decaimento dos autovalores do operador definido por

$$
\mathcal{K}(f)(z)=\int_{X} K(z, w) f(w) d \nu(w), \quad z \in X, \quad f \in L^{2}(X, \nu),
$$

com um núcleo gerador $K$ definido como em 2.0.1), ou seja, núcleos mensuráveis da forma

$$
K(x, y)=\sum_{|\alpha|=0}^{\infty} a_{\alpha} f_{\alpha}(z) \overline{f_{\alpha}(w)}, \quad z, w \in X
$$

sendo $X$ um subconjunto não vazio de $\mathbb{R}^{m+1}$ ou $\mathbb{C}^{m+1}$, munido da medida de Lebesgue $\nu$, e $\left\{f_{\alpha}\right\}$ uma família $L^{2}(X, \nu)$-ortogonal. Suporemos que $K$ pertence à classe $D A I(X)$, 
$\left\{a_{\alpha}\right\} \subset(0, \infty)$ e que

$$
\sum_{\alpha} a_{\alpha}\left\|f_{\alpha}\right\|_{2}<\infty
$$

como na Proposição 2.2.1. Como descrito na Seção 2.3, as hipóteses acima garantem a representação em série

$$
\mathcal{K}(f)=\sum_{\alpha} a_{\alpha}\left\langle f, f_{\alpha}\right\rangle_{2} f_{\alpha}, \quad f \in L^{2}(X, \nu)
$$

com convergência na norma de $L^{2}(X, \nu)$. Ainda, conforme 2.3.3, cada autovalor de $\mathcal{K}$ é dado por

$$
\lambda_{\alpha}(\mathcal{K})=a_{\alpha}\left\|f_{\alpha}\right\|_{2}^{2}, \quad \alpha \in \mathbb{Z}_{+}^{m+1} .
$$

Fixado o contexto acima, a intenção aqui é descrever o decaimento da sequência $\left\{\lambda_{\alpha}(\mathcal{K})\right\}$ via decaimentos adicionais das sequências $\left\{a_{\alpha}\right\}$ e $\left\{\left\|f_{\alpha}\right\|_{2}\right\}$.

Como visto anteriormente, no contexto adotado, já temos garantida a convergência de $\left\{\sum_{|\alpha|=n} \lambda_{\alpha}(\mathcal{K})\right\}$ para 0 , quando $n \rightarrow \infty$, mas não temos garantida a monotonicidade desta mesma sequência. O primeiro resultado do capítulo demanda tal monotonicidade.

Proposição 4.1.1. Suponha que $\left\{\sum_{|\alpha|=n} \lambda_{\alpha}(\mathcal{K})\right\}$ decresce para 0. Se existe um número real positivo $r$ tal que $\left\|f_{\alpha}\right\|_{2}=O\left(|\alpha|^{-r}\right),(|\alpha| \rightarrow \infty)$, então

$$
\sum_{|\alpha|=n} \lambda_{\alpha}(\mathcal{K})=o\left(|\alpha|^{-1-r}\right), \quad(|\alpha| \rightarrow \infty)
$$

Em particular,

$$
\lambda_{\alpha}(\mathcal{K})=o\left(|\alpha|^{-1-r}\right), \quad(|\alpha| \rightarrow \infty) .
$$

Demonstração: Se $\left\|f_{\alpha}\right\|_{2}=O\left(|\alpha|^{-r}\right),(|\alpha| \rightarrow \infty)$, então existe $C>0$ tal que

$$
\left\|f_{\alpha}\right\|_{2} \leq C|\alpha|^{-r}, \quad \alpha \in \mathbb{Z}_{+}^{m+1}
$$

Segue que

$$
n^{r} \sum_{|\alpha|=n} \lambda_{\alpha}(\mathcal{K}) \leq C \sum_{|\alpha|=n} a_{\alpha}\left\|f_{\alpha}\right\|_{2}, \quad n=1,2, \ldots
$$

e, consequentemente,

$$
\sum_{n=0}^{\infty} n^{r} \sum_{|\alpha|=n} \lambda_{\alpha}(\mathcal{K}) \leq C \sum_{n=0}^{\infty} \sum_{|\alpha|=n} a_{\alpha}\left\|f_{\alpha}\right\|_{2}=C \sum_{\alpha} a_{\alpha}\left\|f_{\alpha}\right\|_{2}<\infty .
$$

Uma aplicação do Lema 1.1 .2 produz o resultado final

$$
\sum_{|\alpha|=n} \lambda_{\alpha}(\mathcal{K})=o\left(n^{-1-r}\right), \quad(n \rightarrow \infty) .
$$


Isso conclui a demonstração.

Quando temos disponível algum decaimento para a sequência $\left\{a_{\alpha}\right\}$, então o procedimento utilizado no resultado anterior pode ser adaptado como segue. A hipótese de mononiticidade de $\left\{\sum_{|\alpha|=n} \lambda_{\alpha}(\mathcal{K})\right\}$ pode ser desprezada.

Proposição 4.1.2. Sejam $r$ e s números reais positivos. Se $\left\|f_{\alpha}\right\|_{2}=O\left(|\alpha|^{-r}\right),(|\alpha| \rightarrow$ $\infty)$ e $a_{\alpha}=O\left(|\alpha|^{-s}\right),(|\alpha| \rightarrow \infty)$, então

$$
\sum_{|\alpha|=n} \lambda_{\alpha}(\mathcal{K})=O\left(n^{-2 r-s+m}\right), \quad(n \rightarrow \infty) .
$$

Demonstração: Se os decaimentos para $\left\{a_{\alpha}\right\}_{\alpha}$ e $\left\{\left\|f_{\alpha}\right\|_{2}\right\}_{\alpha}$ descritos no enunciado valem, então podemos deduzir que

$$
\sum_{|\alpha|=n} \lambda_{\alpha}(\mathcal{K})=\sum_{|\alpha|=n} a_{\alpha}\left\|f_{\alpha}\right\|_{2}^{2} \leq C|\alpha|^{-2 r-s} \sum_{|\alpha|=n} 1, \quad n=1,2, \ldots
$$

onde $C>0$. Em outras palavras,

$$
n^{2 r+s} \sum_{|\alpha|=n} \lambda_{\alpha}(\mathcal{K}) \leq C b_{n}^{m+1}, \quad n=1,2, \ldots
$$

onde o número $b_{n}^{m+1}$ é aquele definido no Lema 1.1.6. Como $b_{n}^{m+1}=O\left(n^{m}\right),(n \rightarrow \infty)$, a última desigualdade toma a forma

$$
n^{2 r+s-m} \sum_{|\alpha|=n} \lambda_{\alpha}(\mathcal{K}) \leq C^{\prime}, \quad n=1,2, \ldots,
$$

para algum $C^{\prime}>0$. A demonstração está completa.

Nas condições da proposição acima, podemos estimar diretamente na fórmula $\lambda_{\alpha}(\mathcal{K})=$ $a_{\alpha}\left\|f_{\alpha}\right\|_{2}^{2}, \alpha \in \mathbb{Z}_{+}^{m+1}$, para obter

$$
\lambda_{\alpha}(\mathcal{K})=O\left(|\alpha|^{-2 r-s}\right), \quad(|\alpha| \rightarrow \infty)
$$

\subsection{Decaimento baseado na ordem parcial de $\mathbb{Z}_{+}^{m+1}$}

Nesta seção, manteremos fixadas as hipóteses básicas consideradas no início da seção anterior e suporemos, adicionalmente, que a sequência $\left\{\lambda_{\alpha}(\mathcal{K})\right\}$ decresce para 0 com respeito à ordem parcial previamente fixada. Nestas condições, podemos reindexar a sequência $\left\{\lambda_{\alpha}(\mathcal{K})\right\}$, obtendo uma sequência $\left\{\lambda_{n}(\mathcal{K})\right\}$, de forma que

$$
\lambda_{0}(\mathcal{K}) \geq \lambda_{1}(\mathcal{K}) \geq \lambda_{2}(\mathcal{K}) \geq \cdots \geq 0
$$


Mais ainda, com esta nova notação, podemos organizar os autovalores em blocos:

$$
\begin{aligned}
& \underbrace{\lambda_{1}(\mathcal{K}) \geq \ldots \geq \lambda_{b_{1}^{m+1}}(\mathcal{K})}_{|\alpha|=1} \\
& \underbrace{\lambda_{b_{1}^{m+1}+1}(\mathcal{K}) \geq \ldots \geq \lambda_{b_{1}^{m+1}+b_{2}^{m+1}}(\mathcal{K})}_{|\alpha|=2}, \\
& \underbrace{\lambda_{b_{1}^{m+1}+\ldots+b_{n-1}^{m+1}+1}(\mathcal{K}) \geq \ldots \geq \lambda_{b_{1}^{m+1}+b_{2}^{m+1}+\cdots+b_{n}^{m+1}}(\mathcal{K})}_{|\alpha|=n}, \quad n \geq 1 .
\end{aligned}
$$

Neste sentido, buscamos agora descrever o decaimento da sequência $\left\{\lambda_{n}(\mathcal{K})\right\}$, não mais em termos dos multi-índices. Os resultados a seguir descrevem o decaimento da sequência $\left\{\lambda_{n}(\mathcal{K})\right\}$.

Teorema 4.2.1. Se $\left\|f_{\alpha}\right\|_{2}=O\left(|\alpha|^{-r}\right),(|\alpha| \rightarrow \infty)$, para algum número real positivo $r$, então

$$
\lambda_{n}(\mathcal{K})=o\left(n^{-1-r /(m+1)}\right) \quad(n \rightarrow \infty) .
$$

Demonstração: Em vista de 4.2.1, temos que

$$
\lambda_{b_{n}^{m+1}+\cdots+b_{1}^{m+1}+b_{0}^{m+1}}(\mathcal{K}) \leq \lambda_{\alpha}(\mathcal{K}), \quad|\alpha|=n, \quad n=0,1, \ldots
$$

Esta desigualdade implica que

$$
b_{n}^{m+1} \lambda_{b_{n}^{m+1}+\cdots+b_{1}^{m+1}+b_{0}^{m+1}}(\mathcal{K}) \leq \sum_{|\alpha|=n} \lambda_{\alpha}(\mathcal{K}), \quad n=0,1, \ldots
$$

Se o decaimento do enunciado vale, usando a expressão que define $\lambda_{\alpha}(\mathcal{K})$, deduzimos que existe uma constante $C>0$ tal que

$$
b_{n}^{m+1} n^{r} \lambda_{b_{n}^{m+1}+\cdots+b_{1}^{m+1}+b_{0}^{m+1}}(\mathcal{K}) \leq C \sum_{|\alpha|=n} a_{\alpha}\left\|f_{\alpha}\right\|_{2}, \quad n=0,1, \ldots
$$

Logo, segue que

$$
\sum_{n=0}^{\infty} b_{n}^{m+1} n^{r} \lambda_{b_{n}^{m+1}+\cdots+b_{1}^{m+1}+b_{0}^{m+1}}(\mathcal{K})<\infty .
$$

Devido a 1.1.6 e o comportamento assintótico de $\left\{b_{n}^{m+1}\right\}$ previamente deduzido, obtemos que

$$
\sum_{n=0}^{\infty} n^{r+m} \lambda_{b_{n}^{m+2}}(\mathcal{K})<\infty .
$$

Como $b_{n}^{m+2}=O\left(n^{m+1}\right),(n \rightarrow \infty)$ (veja 2.3 .1$)$, podemos escolher um inteiro positivo $l$ tal que

$$
b_{n}^{m+2} \leq(l n)^{m+1}, \quad n=1,2, \ldots
$$


Retornando a série anterior, como $\left\{\lambda_{n}(\mathcal{K})\right\}$ é decrescente, concluímos que

$$
\sum_{n=0}^{\infty} n^{r+m} \lambda_{(l n)^{m+1}}(\mathcal{K})<\infty .
$$

Disto, podemos escrever que

$$
\sum_{n=0}^{\infty}(\ln )^{r+m} \lambda_{(l n)^{m+1}}(\mathcal{K})<\infty,
$$

e usando novamente o fato de $\left\{\lambda_{n}(\mathcal{K})\right\}$ decrescer, chegamos a

$$
\sum_{n=0}^{\infty}(l n+j)^{r+m} \lambda_{(l n+j)^{m+1}}(\mathcal{K})<\infty, \quad j=0,1, \ldots, l-1 .
$$

Portanto,

$$
\sum_{n=0}^{\infty} n^{r+m} \lambda_{n^{m+1}}(\mathcal{K})<\infty .
$$

Sendo $\left\{\lambda_{n^{m+1}}(\mathcal{K})\right\}$ uma subsequência de uma sequência decrescente, ela mesma decresce para 0. Isso dito, aplicando o Lema 1.1.2 na série anterior, concluímos que

$$
n^{r+m+1} \lambda_{n^{m+1}}(\mathcal{K})=o(1), \quad(n \rightarrow \infty) .
$$

Assim, fixado $\epsilon>0$, existe $N>0$ tal que

$$
\lambda_{n^{m+1}}(\mathcal{K}) \leq \frac{\epsilon}{2^{r+m+1}} \frac{1}{n^{r+m+1}} \quad n=N, N+1, \ldots
$$

Imitando o procedimento utilizado na demonstração do Lema 1.1.4 podemos induzir e chegar a

$$
\lambda_{n^{m+1}+j}(\mathcal{K}) \leq \frac{\epsilon}{\left(n^{m+1}+j\right)^{(r+m+1) /(m+1)}}, \quad n=N, N+1, \ldots,
$$

qualquer que seja o índice $j$ do conjunto $\left\{1,2, \ldots,(n+1)^{m+1}-n^{m+1}-1\right\}$. Mediante os mesmos argumentos utilizados no Lema 1.1.4, concluímos a demostração.

Teorema 4.2.2. Sejam $r$ e s números reais positivos. Se $\left\|f_{\alpha}\right\|_{2}=O\left(|\alpha|^{-r}\right),(|\alpha| \rightarrow \infty)$ e $a_{\alpha}=O\left(|\alpha|^{-s}\right),(|\alpha| \rightarrow \infty)$, então

$$
\lambda_{n}(\mathcal{K})=O\left(n^{-(2 r+s) /(m+1)}\right), \quad(n \rightarrow \infty) .
$$

Demonstração: Suponha que os dois decaimentos iniciais do enunciado valem. Partindo da desigualdade 4.1.2, obtemos

$$
n^{2 r+s} b_{n}^{m+1} \lambda_{b_{n}^{m+2}}(\mathcal{K}) \leq C b_{n}^{m+1}, \quad n=1,2, \ldots,
$$


para algum $C>0$. Segue de 2.3 .2 que

$$
n^{2 r+s} \lambda_{(l n)^{m+1}}(\mathcal{K}) \leq C^{\prime}, \quad n=1,2, \ldots,
$$

para algum $C^{\prime}>0$ e algum inteiro positivo $l$. Uma aplicação do Lema 1.1.4 nos leva a

$$
n^{(2 r+s) /(m+1)} \lambda_{n}(\mathcal{K}) \leq C^{\prime \prime}, \quad n=1,2, \ldots,
$$

para algum $C^{\prime \prime}>0$. A demonstração está completa.

\subsection{Dois exemplos}

Nesta seção, ilustramos os resultados principais do capítulo através de dois exemplos concretos.

No primeiro deles, $X$ é a esfera unitária $\Omega_{m}$ em $\mathbb{C}^{m+1}, m \geq 1, \nu_{m}$ é a medida de probabilidade em $X$ obtida da medida de Lebesgue usual sobre $X$, ou seja, $\nu_{m}(X)=1$, e

$$
f_{\alpha}(z)=z^{\alpha}, \quad \alpha \in \mathbb{Z}_{+}^{m+1} .
$$

Logo, buscamos o decaimento da sequência de autovalores $\left\{\lambda_{\alpha}(\mathcal{K})\right\}$ do operador $\mathcal{K}$ : $L^{2}\left(\Omega_{m}, \nu_{m}\right) \rightarrow L^{2}\left(\Omega_{m}, \nu_{m}\right)$ gerado pelo núcleo

$$
K(z, w)=\sum_{\alpha} a_{\alpha} z^{\alpha} \bar{w}^{\alpha}, \quad z, w \in \Omega_{m},
$$

$\operatorname{com}\left\{a_{\alpha}\right\} \subset(0, \infty)$ satisfazendo $\sum_{\alpha} a_{\alpha}\left\|f_{\alpha}\right\|_{2}<\infty$. Como ([53, p. 16], [63, p. 13])

$$
\int_{\Omega_{m}} z^{\alpha} \bar{z}^{\beta} d \nu_{m}(z)= \begin{cases}0, & \text { se } \alpha \neq \beta \\ \frac{m ! \alpha !}{(m+|\alpha|) !}, & \text { se } \alpha=\beta,\end{cases}
$$

temos que $\left\{f_{\alpha}\right\}_{\alpha}$ é um conjunto $L^{2}\left(\Omega_{m}, \nu_{m}\right)$-ortogonal, $\left\|f_{\alpha}\right\|_{2} \leq 1, \alpha \in \mathbb{Z}_{+}^{m+1}$, e $\sup _{z} \sup _{\alpha}\left|f_{\alpha}(z)\right| \leq 1$. Portanto,

$$
\lambda_{\alpha}(\mathcal{K})=a_{\alpha} \frac{m ! \alpha !}{(m+|\alpha|) !}, \quad \alpha \in \mathbb{Z}_{+}^{m+1} .
$$

Vejamos agora como a sequência $\left\{\left\|f_{\alpha}\right\|_{2}\right\}$ decai. Primeiramente, observe que

$$
\frac{m ! \alpha !}{(m+|\alpha|) !}=\left(\begin{array}{c}
m+|\alpha| \\
|\alpha|
\end{array}\right)^{-1} \frac{\alpha !}{|\alpha| !}=\left(b_{|\alpha|}^{m+1}\right)^{-1} \frac{\alpha !}{|\alpha| !}, \quad \alpha \in \mathbb{Z}_{+}^{m+1} .
$$

Por outro lado, o teorema da aproximação de Stirling nos garante que $|\alpha|^{m}=O\left(b_{|\alpha|}^{m+1}\right)$, $(|\alpha| \rightarrow \infty)$, enquanto que o Lema 1.1 .7 revela que $\alpha ! \leq|\alpha| !, \alpha \in \mathbb{Z}_{+}^{m+1}$. Assim,

$$
\left\|f_{\alpha}\right\|_{2}=O\left(|\alpha|^{-m / 2}\right), \quad(|\alpha| \rightarrow \infty) .
$$


O decaimento na Proposição 4.1.1 toma a forma

$$
\sum_{|\alpha|=n} \lambda_{\alpha}(\mathcal{K})=o\left(|\alpha|^{-1-m / 2}\right) \quad(|\alpha| \rightarrow \infty)
$$

e o da Proposição 4.1 .2 é

$$
\sum_{|\alpha|=n} \lambda_{\alpha}(\mathcal{K})=O\left(|\alpha|^{-s}\right), \quad(|\alpha| \rightarrow \infty)
$$

Quanto aos resultados da Seção 4.2, o decaimento no Teorema 4.2.1 se torna

$$
\lambda_{n}(\mathcal{K})=o\left(n^{-1-m / 2(m+1)}\right), \quad(n \rightarrow \infty),
$$

e aquele do Teorema 4.2 .2 toma a forma

$$
\lambda_{n}(\mathcal{K})=O\left(n^{-1-(s-1) /(m+1)}\right), \quad(n \rightarrow \infty) .
$$

A extensão dos resultados acima para o caso em que $X$ é a bola unitária $\Delta_{m}:=$ $\left\{z \in \mathbb{C}^{m+1}:(z \cdot \bar{z}) \leq 1\right\}$ de $\mathbb{C}^{m+1}$ e $\mu_{m}$ é a medida de probabilidade em $\Delta_{m}$, obtida da medida de Lebesgue usual de $\mathbb{C}^{m+1}$ pode ser alcançada sem muitas dificuldades. Uma relação entre as medidas $\mu_{m}$ e $\nu_{m}$ é dada pela fórmula ([63, p. 9])

$$
\int_{\Delta_{m}} f(w) d \mu_{m}(w)=2(m+1) \int_{0}^{1} r^{2 m+1} \int_{\Omega_{m}} f(r z) d \nu_{m}(z) d r .
$$

Segue que

$$
\begin{aligned}
\int_{\Delta_{m}} \xi^{\alpha} \bar{\xi}^{\beta} d \mu_{m}(\xi) & =2(m+1) \int_{0}^{1} r^{2 m+1+|\alpha|+|\beta|} \int_{\Omega_{m}} z^{\alpha} \overline{z^{\beta}} d \nu_{m}(z) d r \\
& =\frac{2(m+1)}{2(m+1)+|\alpha|+|\beta|} \int_{\Omega_{m}} z^{\alpha} \overline{z^{\beta}} d \nu_{m}(z)
\end{aligned}
$$

Portanto,

$$
\int_{\Delta_{m}} \xi^{\alpha} \bar{\xi}^{\beta} d \mu_{m}(z)= \begin{cases}0, & \text { se } \alpha \neq \beta \\ \frac{(m+1) ! \alpha !}{(m+1+|\alpha|) !}, & \text { se } \alpha=\beta .\end{cases}
$$

Logo,

$$
\left\|\xi^{\alpha}\right\|_{2}^{2}=O\left(|\alpha|^{-m-1}\right), \quad(|\alpha| \rightarrow \infty),
$$

e os decaimentos para este caso podem ser obtidos de maneira análoga ao caso anterior. 



\section{Considerações finais}

O interesse principal deste trabalho foi a obtenção de decaimentos para a sequência de autovalores (valores singulares) do operador integral gerado pelo núcleo $K: X \times X \rightarrow$ $\mathbb{C}$, com representação em série de potências da forma

$$
K(x, y)=\sum_{\alpha} a_{\alpha} x^{\alpha} \bar{y}^{\alpha}, \quad x, y \in X
$$

e pertencente à classe $D A I(X)$, mediante hipóteses razoáveis sobre $X$ e sobre a sequência de coeficientes $\left\{a_{\alpha}\right\}$. Neste capítulo conclusivo, pretendemos destacar algumas informações que não foram discutidas nos resultados incluídos na tese, indicando futuras linhas de atuação envolvendo os objetos aqui abordados.

Os resultados apresentados foram obtidos em um contexto relativamente geral, levando-se em conta a natureza dos núcleos geradores abordados. Esta generalidade dá uma boa margem para investigações adicionais, agora considerando subclasses específicas de núcleos geradores, tendo em vista melhorar alguns dos decaimentos. Por exemplo, em [7] obtivemos estimativas finas para os autovalores de uma certa classe de operadores integrais gerados por núcleos da forma

$$
\sum_{n=0}^{\infty} a_{n}(x \cdot y)^{n}, \quad x, y \in S^{m}
$$

onde $\left\{a_{n}\right\}$ é uma sequência somável de números complexos. Neste caso, as ferramentas da análise esférica entram de forma determinante na obtenção dos resultados e estimativas, devido à natureza especial de tais núcleos. 
No Capítulo 2, em especial na Seção 2.2, apresentamos resultados que tratam da representação em série para certos operadores integrais gerados por núcleos dados por séries de potências satisfazendo algumas hipóteses específicas. Considerando, em especial, o caso em que $X$ é a bola unitária aberta de $\mathbb{R}^{m+1}$ ou $\mathbb{C}^{m+1}$, $\nu$ a medida de Lebesgue usual e a sequência de coeficientes $\left\{a_{\alpha}\right\}$ satisfazendo

$$
\sum_{\alpha}\left|a_{\alpha}\right|\left\|p_{\alpha}\right\|_{2}<\infty
$$

já temos imediatamente que $K \in D A I(X)$, pois $\left\{\left\|p_{\alpha}\right\|_{2}\right\}$ é uniformemente limitada. Como cada conjunto $\left\{\left|x^{\alpha}\right|: x \in X\right\}$ é limitado, a Proposição 2.2.1 nos mostra que

$$
\mathcal{K}(f)(x)=\sum_{\alpha} c_{\alpha}(f) x^{\alpha}, \quad x \in X
$$

$\operatorname{com} c_{\alpha}(f):=a_{\alpha}\left\langle f, p_{\alpha}\right\rangle_{2}, \alpha \in \mathbb{Z}_{+}^{m+1}$. Além disso,

$$
\left|\sum_{\alpha} a_{\alpha}\left\langle f, p_{\alpha}\right\rangle_{2} x^{\alpha}\right| \leq\|f\|_{2} \sum_{\alpha}\left|a_{\alpha}\right|\left\|p_{\alpha}\right\|_{2}<\infty, \quad x \in X, \quad f \in L^{2}(X, \nu) .
$$

Em outras palavras, cada elemento $\mathcal{K}(f)$ da imagem de $\mathcal{K}$ é uma função com representação em série de potências que é absolutamente convergente em $X$. Portanto, a imagem do operador $\mathcal{K}$ contém somente funções analíticas (uma função analítica é entendida aqui, como aquela definida em um aberto, coincidindo com sua expansão de Taylor em torno de cada ponto de seu domínio). Note ainda que, da equação 3.2.3 e da fórmula (4.3.1), obtemos as seguintes condições suficientes para que a imagem do operador $\mathcal{K}$ em questão possua somente funções analíticas (aqui $b_{n}=\sum_{|\alpha|=n}\left|a_{\alpha}\right|$, $n=0,1, \ldots)$ :

$$
\sum_{n=0}^{\infty} b_{n} n^{-(2+m) / 4}<\infty \quad \text { e } \quad \sum_{n=0}^{\infty} b_{n} n^{-(m+1) / 2}<\infty,
$$

respectivamente. Esta propriedade é uma consequência do fato que uma função, descrita por uma série de potências e definida em seu domínio de convergência, é analítica ([4, 37, 53]).

Quando cada coeficiente $a_{\alpha}$ é um número real positivo, o núcleo $K$ é positivo definido, ou seja,

$$
\sum_{i, j=1}^{n} \overline{c_{i}} c_{j} K\left(x_{i}, x_{j}\right) \geq 0
$$

qualquer que seja $n \geq 1,\left\{x_{1}, x_{2}, \ldots, x_{n}\right\} \subset X$ e $\left\{c_{1}, c_{2}, \ldots, c_{n}\right\} \subset \mathbb{C}$. Podemos então considerar o espaço de Hilbert de reprodução $\mathcal{H}_{K}$ de $K([3])$, ou seja, o completamento 
do espaço vetorial gerado pelo conjunto $\left\{K_{z}:=K(z, \cdot), z \in X\right\}$ munido do produto interno $\langle\cdot, \cdot\rangle_{K}$, dado pela fórmula

$$
\left\langle K_{z}, K_{w}\right\rangle_{K}=K(z, w), \quad z, w \in X
$$

Uma de suas principais atribuições é reproduzir seus elementos através da igualdade

$$
f(z)=\left\langle K_{z}, f\right\rangle_{K}, \quad z \in X, \quad f \in \mathcal{H}_{K}
$$

Como consequência da Proposição 3.2.6 de [17], a imagem do operador $\mathcal{K}$ torna-se um subespaço de $\mathcal{H}_{K}$. Mais ainda, quando $K \in D A I(X)$, temos que ([17, p. 47])

$$
\langle\mathcal{K}(f), g\rangle_{K}=\langle f, g\rangle_{2}, \quad f \in L^{2}(X, \nu), \quad g \in \mathcal{H}_{K}
$$

Consequentemente, a imagem de $\mathcal{K}$ é um subespaço denso de $\mathcal{H}_{K}$, pois o complemento ortogonal dela em $\mathcal{H}_{k}$ é o subespaço nulo.

Desta forma, um problema interessante a ser considerado é estudar propriedades do espaço $\mathcal{H}_{K}$, a partir das condições assumidas ou outras que envolvam a sequência de coeficientes $\left\{a_{\alpha}\right\}$. Por exemplo, questões sobre a analiticidade das funções de $\mathcal{H}_{K}$ ou estimativas para os números de recobrimento do conjunto $\left\{f \in \mathcal{H}_{K}:\|f\|_{K} \leq 1\right\}$. Lembramos que, dado um subconjunto $A$ de um espaço métrico $M$, o número de recobrimento $\mathcal{N}(\eta, A, M)$ é definido como o número mínimo de bolas de raio $\eta$ em $M$, necessárias para cobrir o conjunto $A$. As referências [16, 40, 61, 62, 59], além de delinear claramente a necessidade de se estudar tais números, apresentam resultados interessantes sobre o tema. 



\section{Referências Bibliográficas}

[1] Abramowitz, M.; Stegun, I. A., Handbook of mathematical functions with formulas, graphs, and mathematical tables. National Bureau of Standards Applied Mathematics Series, Dover, New York, 1964.

[2] Andrews, G. E.; Askey, R.; Roy, R., Special functions. Encyclopedia of Mathematics and its Applications, 71. Cambridge University Press, Cambridge, 1999.

[3] Aronszajn, N., Theory of Reproducing Kernels. Trans. Amer. Math. Soc., 68 (1950), no. $3,337-404$.

[4] Axler, S.; Bourdon, P.; Ramey, W., Harmonic function theory. Second edition. Graduate Texts in Mathematics, 137. Springer-Verlag, New York, 2001.

[5] Azevedo, D.; Menegatto, V. A., Eigenvalue decay of integral operators generated by power series-like kernels. Math. Inequal. Appl., a aparecer.

[6] Azevedo, D.; Menegatto, V. A., Estimates for singular values of integral operators generated by power series kernels on the sphere. Submetido para publicação.

[7] Azevedo, D.; Menegatto V. A., Sharp estimates for eigenvalues of integral operators generated by dot product kernels on the sphere. Submetido para publicação.

[8] Baker, J. S., Integration over spheres and the divergence theorem for balls. Amer. Math. Monthly, 104 (1997), no. 1, 36-47.

[9] Berg, C.; Christensen, J. P. R.; Ressel, P., Harmonic analysis on semigroups: Theory of positive definite and related functions, Springer-Verlag, 1984.

[10] Buescu, J.; Paixão, A. C., Eigenvalue distribution of Mercer-like kernels. Math. Nachr. 280 (2007), no. 9-10, 984-995. 
[11] Buescu, J.; Paixão, A. C., Eigenvalues of positive definite integral operators in unbounded intervals. Positivity, 10 (2006), 627-646.

[12] Castro, M. H.; Menegatto, V. A., Eigenvalue decay of positive integral operators on the sphere. Math. Comp. 81 (2012), no. 280, 2303-2317.

[13] Cobos, F.; Kühn, T., Eigenvalues of integral operators with positive definite kernels satisfying integrated Hölder conditions over metric compacta. J. Approx. Theory, 63 (1990), 39-55.

[14] Cochran, J. A.; Lukas, M. A., Differentiable positive definite kernels and Lipschitz continuity. Math. Proc. Camb. Phil. Soc., 104 (1988), 361-369.

[15] Cucker, F.; Smale, S., On the mathematical foundations of learning. Bull. Amer. Math. Soc. (New Series), 39 (2001), no. 1, 1-49.

[16] Donoghue, W. F. Jr., Reproducing kernel spaces and analytic continuation. Rocky Mountain Journal of Mathematics., 10 (1980), no. 1, 85-97.

[17] Ferreira, J. C., Operadores integrais positivos e espaços de Hilbert de reprodução. Tese de doutorado, ICMC-USP, 2011.

[18] Ferreira, J. C.; Menegatto V. A., Eigenvalues of integral operators defined by smooth positive definite kernels. Integral Equations Operator Theory, 64 (2009), no. $1,61-81$.

[19] Ferreira, J. C.; Menegatto, V. A.; Oliveira, C. P., On the nuclearity of integral operators. Positivity, 13 (2009), no. 3, 519-541.

[20] Ferreira, J. C.; Menegatto, V. A.; Peron, A. P., Integral operators on the sphere generated by positive definite smooth kernels. J. Complexity, 24 (2008), no. 5-6, 632-647.

[21] Folland, G. B., Real analysis: Modern techniques and their applications, John Wiley \& Sons, 1999.

[22] Folland, G. B., How to Integrate a polynomial over a sphere. The American Mathematical Monthly, 108 (2001), no. 5, 446-448.

[23] Fredholm, I., Sur une classe d'équations fonctionnelles, Acta Math., 27 (1903), no. 1, 365-390. 
[24] Gel'fond, A. O., On estimation of certain determinants and the application of these estimations to the distribution of eigenvalues. Amer. Math. Soc. Transl. (2) 12 (1959), 163-179.

[25] Gohberg, I.; Gohberg, S.; Krupnik, N., Traces and determinants of linear operators, Operator Theory: Advances and Applications, 116, Birkhäuser Verlag, Basel, 2000.

[26] Groemer, H., Geometric applications of Fourier series and spherical harmonics. (English summary) Encyclopedia of Mathematics and its Applications, 61. Cambridge University Press, Cambridge, 1996.

[27] Grothendieck, A., La théorie de Fredholm. Bull. Soc. Math. France, 84 (1956), 319-384.

[28] Hildebrandt, T. H., On unconditional convergence in normed vector spaces. Bull. Amer. Math. Soc., 46 (1940), no. 12, Part 1, 959-962.

[29] Hille, E.; Tamarkin, J. D., On the characteristic values of linear integral equations. Acta Math. 57 (1931), no. 1, 1-76.

[30] Han, Y. B, Eigenvalues of higher-dimensional positive definite kernels. Acta Math. Sinica, 36 (1993), 188-194.

[31] Jordão, T.; Menegatto, V. A., Integral operators generated by multi-scale kernels. J. Complexity, 26 (2010), 187-199.

[32] Jordão, T., Diferenciabilidade em espaços de Hilbert de reprodução sobre a esfera. Tese de doutorado, ICMC-USP, 2012.

[33] Knopp, K., Infinite sequences and series. Translated by Frederick Bagemihl. Dover Publications, Inc., New York, 1956.

[34] König, H., Eigenvalues of operators and applications. In Handbook of the geometry of Banach spaces, Vol. I, North-Holland, Amsterdam, (2001), 941-974.

[35] König, H., Eigenvalue distribution of compact operators. Operator Theory: Advances and Applications, 16. Birkhäuser Verlag, Basel, 1986.

[36] Kotljar, B. D., Singular numbers of integral operators. Differentsial'nye Uravneniya 14 (1978), no. 8, 1473-1477. 
[37] Krantz, S. G.; Parks, H. R., A primer of real analytic functions. Second edition. Birkhäuser Advanced Texts: Basler Lehrbücher. [Birkhäuser Advanced Texts: Basel Textbooks] Birkhäuser Boston, Inc., Boston, MA, 2002.

[38] Kühn, T., Eigenvalues of integral operators generated by positive definite Hölder continuous kernels on metric compacta. Indag. Math., 49 (1987), no. 1, 51-61.

[39] Kühn, T., Eigenvalues of integral operators with smooth positive definite kernels. Arch. Math. (Basel), 49 (1987), no. 6, 525-534.

[40] Kühn, T., Covering numbers of Gaussian reproducing kernel Hilbert spaces. $J$. Complexity 27 (2011), 489-499.

[41] Little, G., Eigenvalues of positive power series kernels. Bull. London Math. Soc. 28 (1996), no. 1, 43-50.

[42] Little, G., Eigenvalues of positive integral operators with certain entire kernels. Math. Proc. Camb. Phil. Soc 99 (1986), 535-545

[43] Little, G.; Reade, J.B., Eigenvalues of analytic kernels. SIAM J. Math. Anal. 15 (1984), 132-136.

[44] Lu, F.; Sun, H., Positive definite dot product kernels in learning theory. Adv. Comput. Math. 22 (2005), 181-198.

[45] Mercer, J., Functions of positive and negative type and their connection with the theory of integral equations. Philos. Trans. R. Soc. Lond. Ser. A, 209 (1909), $415-446$.

[46] Minh. H. Q., Some properties of Gaussian reproducing kernel Hilbert spaces and their implications for function approximation and learning theory. Constr. Approx., 32 (2010), no. 2, 307-338.

[47] Pietsch, A., Nuclear locally convex spaces, Ergebnisse Der Mathematick und Ihrer Grensgebiete, Volume 66, Springer-Verlag, New York/Heidleberg/Berlin, 1972

[48] Reade, J., Eigenvalues of positive definite kernels. SIAM J. Math. Anal., 14 (1983), no. $1,135-140$.

[49] Reade, J., Eigenvalues of positive definite kernels II. SIAM J. Math. Anal., 15 (1984), no. 1, 137-142. 
[50] Reade, J., On the sharpness of Weyl's estimate for eigenvalues of smooth kernels. SIAM J. Math. Anal., 16 (1985), no. 3, 137-142.

[51] Reade, J., Eigenvalues of smooth positive definite kernels. Proc. of the Edinburg Math. Soc, 19 (1992), no. 35, 41-45.

[52] Royden, L. H., Real Analysis, Second edition, The Macmillan Company, 1968.

[53] Rudin, W., Function theory in the unit ball of $\mathbb{C}^{n}$. Reprint of the 1980 edition. Classics in Mathematics. Springer-Verlag, Berlin, 2008.

[54] Saitoh, S., Integral transforms, reproducing kernels and their applications, Longman, Gunna University, Japan, 1997.

[55] Schaback, R., Multivariate interpolation by polynomials and radial basis functions. Constr. Approx. 21 (2005), 293-317.

[56] Smale, S.; Zhou, D. X., Learning theory estimates via integral operators and their approximations. Constr. Approx., 26 (2007), 153-172.

[57] Smola, A. J.; Ovári, Z. L.; Williamson, R. C., Regularization with Dot-Product Kernels. In Proc. of the Neural Information Processing Systems (NIPS), 308-314, MIT-Press, 2000.

[58] Sun, H.; Wu, Q., Application of integral operator for regularized least-square regression. Math. Comput Modelling, 49 (2009), 276-285.

[59] Sun, H. W; Zhou, D. X., Reproducing kernel Hilbert spaces associated with analytic translation-invariant Mercer kernels. J. Fourier Anal. Appl. 14 (2008), no.1, 89-101.

[60] Weidmann, J., Linear operators in Hilbert spaces. Springer-Verlag, 1980.

[61] Zhou, D. X., Capacity of reproducing kernel spaces in learning theory. IEEE Transactions on information theory, 49 (2003), no. 7, 1743-1752.

[62] Zhou, D. X.; The covering number in learning theory. J. Complexity, 18 (2002), 739-767.

[63] Zhu, K., Spaces of holomorphic functions in the unit ball. Graduate Texts in Mathematics, 226. Springer-Verlag, New York, 2005.

[64] Zwicknagl, B., Power Series Kernels. Constr. Approx. 29 (2009), no. 1, 61-84. 


\section{Índice Remissivo}

$(\cdot), 6$

$D A I(X), 16$

$L^{2}(X, \mu)$-ortogonal, 22

$\mathcal{K}, 13$

$\langle\cdot, \cdot\rangle_{2}, 7$

$\mathbb{Z}_{+}, 4$

$\preceq, 15$

$\left\{a_{\alpha}\right\}, 16$

$\left\{b_{n}\right\}, 26$

$a_{n}=O\left(b_{n}\right), 1$

$a_{n}=o\left(b_{n}\right), 2$

$a_{n} \sim b_{n}, 2$

$b_{k}^{m+1}, 4$

$p_{\alpha}, 9$

$s_{n}, 12$

$x^{\alpha}, 8$

Função analítica, 48

Desigualdade

de Hölder, 7

de multi-índices, 5

Espaço de Hilbert de reprodução, 48

Espaço de medida, 7

Medida de probabilidade, 44

Monômio, 8

Multi-índices, 4
Núcleo, 13

diagonalmente absolutamente integrável, 16

Série de potências, 15

Operador

integral, 13

nuclear, 23

operadores

compactos, 10

positivos, 11

Teorema

da aproximação de Stirling, 4

de Fubini-Tonelli, 7

de Hilbert-Schmidt, 11

Multinomial, 5

Valores singulares, 12 